\title{
An empirical Bayesian solution to the source reconstruction problem in EEG
}

\author{
Christophe Phillips, ${ }^{\mathrm{a}, *}$ Jeremie Mattout, ${ }^{\mathrm{b}}$ Michael D. Rugg, ${ }^{\mathrm{c}}$ Pierre Maquet, ${ }^{\mathrm{a}}$ and Karl J. Friston ${ }^{\mathrm{b}}$ \\ ${ }^{a}$ Centre de Recherches du Cyclotron, B30, Université de Liège, Liège 4000, Belgium \\ ${ }^{\mathrm{b}}$ Wellcome Department of Imaging Neuroscience, Institute of Neurology, University College London, London, UK \\ ${ }^{c}$ Center for the Neurobiology of Learning and Memory, University of California, Irvine, CA 92717, USA
}

Received 8 April 2004; revised 23 September 2004; accepted 21 October 2004

Available online 5 January 2005

\begin{abstract}
Distributed linear solutions of the EEG source localisation problem are used routinely. In contrast to discrete dipole equivalent models, distributed linear solutions do not assume a fixed number of active sources and rest on a discretised fully $3 \mathrm{D}$ representation of the electrical activity of the brain. The ensuing inverse problem is underdetermined and constraints or priors are required to ensure the uniqueness of the solution. In a Bayesian framework, the conditional expectation of the source distribution, given the data, is attained by carefully balancing the minimisation of the residuals induced by noise and the improbability of the estimates as determined by their priors. This balance is specified by hyperparameters that control the relative importance of fitting and conforming to various constraints. Here we formulate the conventional "Weighted Minimum Norm" (WMN) solution in terms of hierarchical linear models. An "Expectation-Maximisation" (EM) algorithm is used to obtain a "Restricted Maximum Likelihood" (ReML) estimate of the hyperparameters, before estimating the "Maximum a Posteriori" solution itself. This procedure can be considered a generalisation of previous work that encompasses multiple constraints. Our approach was compared with the "classic" WMN and Maximum Smoothness solutions, using a simplified 2D source model with synthetic noisy data. The ReML solution was assessed with four types of source location priors: no priors, accurate priors, inaccurate priors, and both accurate and inaccurate priors. The ReML approach proved useful as: (1) The regularisation (or influence of the a priori source covariance) increased as the noise level increased. (2) The localisation error (LE) was negligible when accurate location priors were used. (3) When accurate and inaccurate location priors were used simultaneously, the solution was not influenced by the inaccurate priors. The ReML solution was then applied to real somatosensory-evoked responses to illustrate the application in an empirical setting.

(C) 2004 Elsevier Inc. All rights reserved.
\end{abstract}

Keywords: EEG; Restricted maximum likelihood (ReML) solution; Expectation-maximisation (EM) procedure; Source reconstruction; Distributed solution

\footnotetext{
* Corresponding author. Fax: +32 43662946.

E-mail address: c.phillips@ulg.ac.be (C. Phillips).

Available online on ScienceDirect (www.sciencedirect.com).
}

\section{Introduction}

The problem of recovering volume current sources from superficial electromagnetic measurement is intrinsically ill-posed (von Helmholtz and Hermann, 1853) and the spatial configuration of neuronal activity cannot be determined uniquely, based on EEG and/or MEG recordings alone (Nunez, 1981). To resolve the nonuniqueness of this inverse problem, assumptions about the solution must be made to obtain a unique and "optimal" solution according to the criteria employed.

Two approaches are generally used to solve this inverse problem: "Equivalent Current Dipole" (ECD) approaches, where the EEG/MEG signals are assumed to be generated by a relatively small number of focal sources (Aine et al., 2000; Miltner et al., 1994; Scherg and Ebersole, 1994; Scherg et al., 1999), and the "Distributed Linear" (DL) approach, where "all" possible source locations are considered simultaneously (Backus and Gilbert, 1970; Grave de Peralta Menendez and Gonzalez Andino, 1999; Hämäläinen and Ilmoniemi, 1994; Pascual-Marqui, 1999; Sarvas, 1987; Uutela et al., 1999).

In Phillips et al. (2002a), we introduced a solution based on the "Weighted Minimum L2-Norm" (WMN) solution where the source space was modelled by spatially "Informed Basis Functions" (IBF): The activity over the sources is defined in terms of a linear combination of orthogonal basis functions. This approach can be seen as a compromise between the DL solution where all the dipoles are independent, and the ECD solution where only a limited set of sources can be active. Indeed, the ECD approach can be understood as using spatial IBF, where the spatial support of the basis functions is limited to a single point.

Having specified a suitable basis set, further constraints on the sources can be introduced in a probabilistic (Bayesian) sense. The major problem here is the introduction of multiple constraints and their appropriate weighting, while accounting for observation noise (Gonzalez Andino et al., 2001). In Phillips et al. (2002b), we introduced a simple "Restricted Maximum Likelihood" (ReML) procedure to estimate a single hyperparameter, i.e., balance between fitting the data and conforming to the priors. Here we reformulate the 
WMN solution in terms of a hierarchical linear model. With this approach, any number of constraints (or priors) on the source or noise covariance matrices can be introduced. An "ExpectationMaximisation" (EM) algorithm is used to obtain a ReML estimate of the hyperparameters associated with each constraint. This enables the "Maximum a posteriori" solution to be calculated.

This paper is divided into two subsequent sections. In the first section ("Theory"), the theoretical background and operational details of our approach are described. The first part ("Weighted minimum norm and Bayesian solutions") introduces the WMN solution in a Bayesian framework, while the second ("Hierarchical parametric empirical Bayes approach" and "Restricted maximum likelihood solution") introduces the hierarchical "Parametric Empirical Bayes" (PEB) and ReML approach. "Spatial constraints and priors" section is a brief synopsis of the technique presented in full in Phillips et al. (2002b) defining the constraints used. In the last section ("Applications"), we use a simplified model and simulated signal (with added noise) to explore the behaviour of our approach over a range of variables and noise levels ("Simulations with a simplified model"). To assess the construct validity of our method, we compare it with two established approaches to the source localisation problem: simple WMN and Maximum Smoothness (MS, LORETA-like) solutions. Finally, in "Real source reconstruction" our approach is applied to real somatosensory ERP data, with a realistic head model based on the anatomy of the subject.

In the rest of this paper, $a, \vec{a}, \mathbf{a}$ and $\mathbf{A}$ will represent, respectively, a scalar, a vector of size $3 \times 1$, a vector of any size $N_{a} \times 1$ and a matrix; $\mathbf{A}^{t}$ will designate the transpose of $\mathbf{A}$.

\section{Theory}

The instantaneous source localisation problem in EEG can be summarised by the following equation:

$\mathbf{v}=\mathcal{F}(\vec{r}, \vec{j})+\varepsilon$

where $\mathbf{v}$, a vector of size $N_{e} \times 1$, is the potential at the $N_{e}$ electrodes; $\vec{r}$ and $\vec{j}$ are the source location and moment; $\varepsilon$ is the additive noise; and $\mathcal{F}$ is the function linking the source $(\vec{r}, \vec{j})$ and the potential $\mathbf{v}$. The function $\mathcal{F}$ is the solution of the forward problem and depends only on the head model adopted (conductivity and spatial configuration).

For $N_{d}$ sources defined by $\overrightarrow{r_{i}}$ and $\overrightarrow{j_{i}}$ (with $i=1, \ldots, N_{d}$ ), the source localisation problem (1) is rewritten, thanks to the superposition theorem, as:

$\mathbf{v}=\sum_{i=1}^{N_{d}} \mathcal{F}\left(\overrightarrow{r_{i}}, \overrightarrow{j_{i}}\right)+\varepsilon$

In this paper, the sources of the EEG signal are modelled by a fixed three-dimensional grid of current dipoles throughout the brain volume, conforming to the "Distributed Linear Solution" approach where $N_{d}$ is much larger than $N_{e}$. Because the location $\overrightarrow{r_{i}}$ of each current source is now fixed, Eq. (1) becomes an underdetermined but linear problem:

$\mathbf{v}=\mathbf{L} \mathbf{j}+\varepsilon$

where $\mathbf{j}$ is a vector representing the current dipoles at all the $N_{d}$ locations simultaneously, and $\mathbf{L}$ is the lead field matrix linking the source amplitudes $\mathbf{j}$ to the electrical potential $\mathbf{v}$. If the source orientation is left free, then $\mathbf{j}=\left[\vec{j}_{1}^{t} \vec{j}_{2}^{t} \ldots \vec{j}_{N_{d}}\right]^{t}$, where $\overrightarrow{j_{i}}=\left[j_{x, i}\right.$ $\left.j_{y, i} j_{z, i}\right]^{t}$ encodes both orientation and amplitude of the $i$ th current dipole. Otherwise, for orientated sources, $\mathbf{j}=\left[j_{1} j_{2} \ldots j_{N_{d}}\right]^{t}$, where each $j_{i}$ specifies only the amplitude of the $i^{\text {th }}$ current dipole.

For discrete data time series with $N_{t}$ time bins, Eq. (3) can be expressed as a multivariate linear model:

$\mathbf{V}=\mathbf{L} \mathbf{J}+\boldsymbol{\varepsilon}$

with $\mathbf{V}=\left[\mathbf{v}_{1} \mathbf{v}_{2} \ldots \mathbf{v}_{N_{t}}\right], \mathbf{J}=\left[\mathbf{j}_{1} \mathbf{j}_{2} \ldots \mathbf{j}_{N_{t}}\right]$ and $\boldsymbol{\varepsilon}=\left[\boldsymbol{\varepsilon}_{1} \boldsymbol{\varepsilon}_{2} \ldots \boldsymbol{\varepsilon}_{N_{t}}\right]$ where $\mathbf{v}_{l}, \mathbf{j}_{l}$ and $\boldsymbol{\varepsilon}_{l}$ are the potential, current dipoles and additive noise at the $l^{\text {th }}$ time instant.

\section{"Weighted minimum norm" and Bayesian solutions}

As stated in the Introduction, the source localisation problem is intrinsically ill-posed. With the DL approach, we face the linear but largely under-determined problem expressed in Eqs. (3) or (4). One common approach to solve this problem is the "Weighted Minimum Norm" (WMN) solution or Tikhonov regularisation method (Tikhonov and Arsenin, 1977), where the a priori constraints can be interpreted in a Bayesian sense.

The WMN solution constrains the reconstructed source distribution by minimising a linear mixture of some weighted norm $\|\mathbf{H j}\|$ of the source amplitudes $\mathbf{j}$ and the residuals of the fit. Assuming the noise component $\varepsilon$ is Gaussian $\varepsilon \sim \mathcal{N}\left(0, \mathbf{C}_{\varepsilon}\right)$ with a known covariance matrix $\mathbf{C}_{\varepsilon}$, the regularised problem is expressed as:

$\hat{\mathbf{j}}=\arg \min _{j}\left\{\left\|\mathbf{C}_{\varepsilon}^{-1 / 2}(\mathbf{L j}-\mathbf{v})\right\|^{2}+\lambda\|\mathbf{H} \mathbf{j}\|^{2}\right\}$

or

$\hat{\mathbf{j}}=\arg \min _{j}\left\{(\mathbf{L} \mathbf{j}-\mathbf{v})^{t} \mathbf{C}_{\varepsilon}^{-1}(\mathbf{L} \mathbf{j}-\mathbf{v})+\lambda \mathbf{j}^{t}\left(\mathbf{H}^{t} \mathbf{H}\right) \mathbf{j}\right\}$

where the hyperparameter $\lambda$ expresses the balance between fitting the model $\left\|\mathbf{C}_{\varepsilon}^{-1 / 2}(\mathbf{L j}-\mathbf{v})\right\|$ and minimising the a priori constraint $\|\mathbf{H j}\|$.

The solution of Eqs. (5a) and (5b) for a given $\lambda$ is:

$\hat{\mathbf{j}}=\mathbf{T v}$

where

$\mathbf{T}=\left[\mathbf{L}^{t} \mathbf{C}_{\varepsilon}^{-1} \mathbf{L}+\lambda\left(\mathbf{H}^{t} \mathbf{H}\right)\right]^{-1} \mathbf{L}^{t} \mathbf{C}_{\varepsilon}^{-1}$

$\mathbf{T}=\left(\mathbf{H}^{t} \mathbf{H}\right)^{-1} \mathbf{L}^{t}\left[\mathbf{L}\left(\mathbf{H}^{t} \mathbf{H}\right)^{-1} \mathbf{L}^{t}+\lambda \mathbf{C}_{\varepsilon}\right]^{-1}$

using the matrix inversion Lemma.

The important and useful connection with Bayesian estimates of the sources rests on Gaussian assumptions, when the conditional expectation or posterior mean of the source amplitudes $\mathbf{j}$ is given by:

$E(\mathbf{j} \mid \mathbf{v})=\left[\mathbf{L}^{t} \mathbf{C}_{\varepsilon}^{-1} \mathbf{L}+\mathbf{C}_{\mathbf{j}}^{-1}\right]^{-1} \mathbf{L}^{t} \mathbf{C}_{\varepsilon}^{-1} \mathbf{v}$

$E(\mathbf{j} \mid \mathbf{v})=\mathbf{C}_{\mathbf{j}} \mathbf{L}^{t}\left[\mathbf{L} \mathbf{C}_{\mathbf{j}} \mathbf{L}^{t}+\mathbf{C}_{\varepsilon}\right]^{-1} \mathbf{v}$ 
where $\mathbf{C}_{\mathbf{j}}$ is the prior covariance of the sources. Comparing Eqs. (8a) and (8b) with Eqs. (7a) and (7b) provides the motivation for choosing forms of $\mathbf{H}$, where

$$
\lambda\left(\mathbf{H}^{t} \mathbf{H}\right)=\mathbf{C}_{\mathbf{j}}^{-1}
$$

Solutions (7a) (7b) (8a) (8b) rely on the precise knowledge of $\mathbf{C}_{\varepsilon}$ and $\mathbf{C}_{\mathbf{j}}$ and, for the case where $\mathbf{C}_{\mathbf{j}}^{-1}$ tends to zero (flat priors), they represent the "Maximum Likelihood solution"; see Appendix A.1.

In Eqs. (5a) and (5b), a single constraint $\mathbf{H}$ was employed. One could generalise this approach to include a whole series of constraints or priors $\mathbf{H}_{1}, \mathbf{H}_{2}, \ldots$, leading to the "generalised (weighted) minimum norm" (gWMN):

$$
\begin{aligned}
\hat{\mathbf{j}}= & \operatorname{argmin}\left\{\left\|\mathbf{C}_{\varepsilon}^{-1 / 2}(\mathbf{L} \mathbf{j}-\mathbf{v})\right\|^{2}+\lambda_{1}\left\|\mathbf{H}_{1} \mathbf{j}\right\|^{2}\right. \\
& \left.+\lambda_{2}\left\|\mathbf{H}_{2} \mathbf{j}\right\|^{2}+\lambda_{3}\left\|\mathbf{H}_{3} \mathbf{j}\right\|^{2}+\ldots\right\}
\end{aligned}
$$

or

$$
\begin{aligned}
\hat{\mathbf{j}}= & \operatorname{argmin}\left\{(\mathbf{L} \mathbf{j}-\mathbf{v})^{t} \mathbf{C}_{\varepsilon}^{-1}(\mathbf{L} \mathbf{j}-\mathbf{v})\right. \\
& \left.+\mathbf{j}^{t}\left(\lambda_{1} \mathbf{R}_{1}+\lambda_{2} \mathbf{R}_{2}+\lambda_{3} \mathbf{R}_{3}+\ldots\right) \mathbf{j}\right\}
\end{aligned}
$$

with $\mathbf{R}_{k}=\mathbf{H}_{k}^{t} \mathbf{H}_{k}$. As in Eq. (9), we also assume that the source amplitudes $\mathbf{j}$ follow a Gaussian distribution with zero mean:

$\mathbf{j} \propto \mathcal{N}\left(O, \mathbf{C}_{\mathbf{j}}\right)$

where the precision matrix $\mathbf{C}_{\mathbf{j}}^{-1}$ (precision is the inverse of variance) is expressed in terms of a linear basis set, specified by $\mathbf{R}_{i}$ :

$\mathbf{C}_{\mathbf{j}}^{-1}=\lambda_{1} \mathbf{R}_{1}+\lambda_{2} \mathbf{R}_{2}+\lambda_{3} \mathbf{R}_{3}+\ldots$

With the gWMN approach, the precision of the source distribution $\mathbf{j}$ can thus be modelled via a set of a priori defined basis precisions. By increasing the precision at some locations, the corresponding sources are forced to be closer to zero. In some instances, the priors can be so precise that they preclude the solution from spanning certain subspaces of the solution space, i.e., if $\mathbf{C}_{\mathbf{j}}$ was 0 somewhere along its leading diagonal, the regularisation or penalty would be infinite at that location and the conditional estimate (8a) and (8b) would be zero. For example, the prior variance of source activity in white matter can be set to 0 . In these situations, it is computationally more efficient to remove these "impossible" subspaces before computing the WMN solution.

The WMN solution (6) depends on the hyperparameter $\lambda$. The hyperparameter $\lambda$ balances the relative contribution of fitting the model $\mathbf{C}_{\varepsilon}^{-1 / 2}(\mathbf{L j}-\mathbf{v})$ (or likelihood of the data) and the constraint on the solution $\mathbf{H j}$ (or a priori on the data). As $\lambda$ varies, the regularised solution $\hat{\mathbf{j}}_{\lambda}$ changes in a way that depends on $\lambda$. Therefore, the choice of $\lambda$ is crucial. As a general rule, the degree of regularisation (expressed by $\lambda$ ) should increase with the level of noise in the data, i.e., the importance of the priors should increase as the model fit decreases, but this rule is not sufficient to estimate $\lambda$.

A heuristic way to display and understand the properties of $\hat{\mathbf{j}}_{\lambda}$ is to plot the (weighted) norm of the regularised solution $\left\|\mathbf{H} \hat{\mathbf{j}}_{\lambda}\right\|^{2}$, versus the norm of the residual vector $\left\|\mathbf{C}_{\varepsilon}^{-1 / 2}\left(\mathbf{L} \hat{\mathbf{j}}_{\lambda}-\mathbf{v}\right)\right\|^{2}$ for different values of $\lambda$. The curve obtained usually has an $L$ shape (in ordinary or double logarithmic scale), hence its name "L-curve". A satisfactory $\lambda$ would lie close to the inflection of the L-curve (Hansen, 1992). A major disadvantage of the L-curve approach is that the solution must be calculated for a large number of values of $\lambda$ to find an appropriate regularisation level. Moreover, this Lcurve approach cannot be extended to estimate multiple hyperparameters such as in Eqs. (10a), (10b) and (12). This would require an extensive search in the hyperparameter space to determine the inflection in a hyperplane (Brooks et al., 1999).

In Phillips et al. (2002b), we introduced an iterative procedure to estimate $\lambda$ while calculating $\hat{\mathbf{j}}$ for the simple case of one hyperparameter. In the following section, we extend this approach to solve the generalised WMN problems (10a) and (10b) with multiple hyperparameters thereby allowing noise to be accommodated by balancing the likelihood and priors of the data, and determining the relative contribution of different priors on the data.

\section{Hierarchical parametric empirical Bayes approach}

The source localisation problems (3) or (4) can be expressed in the context of a two-level hierarchical "Parametric Empirical Bayes" (PEB) model, by

$\left\{\begin{array}{ccc}\mathbf{v}=\mathbf{L j} & +\varepsilon_{1} \\ \mathbf{j}=0 & + & \varepsilon_{2}\end{array}\right.$

where both $\varepsilon_{1}$ and $\varepsilon_{2}$ follow a Gaussian distribution with zero mean:

$\varepsilon_{1} \propto \mathcal{N}\left(0, \mathbf{C}_{\varepsilon}\right)$

$\varepsilon_{2} \propto \mathcal{N}\left(0, \mathbf{C}_{\mathbf{j}}\right)$

Within this framework, the covariance matrices $\mathbf{C}_{\varepsilon}$ and $\mathbf{C}_{\mathbf{j}}$, which are equivalent to those in Eqs. (5a) (5b) (6) (7a) (7b) (8a) (8b) (9) (10a) (10b) (11) (12), can be modelled as a linear combination of covariance components:

$\mathbf{C}_{\varepsilon}=\mu_{1} \mathbf{C}_{e_{1}, 1}+\mu_{2} \mathbf{C}_{e_{1}, 2}+\ldots$

$\mathbf{C}_{\mathbf{j}}=v_{1} \mathbf{C}_{e_{2}, 1}+v_{2} \mathbf{C}_{e_{2}, 2}+\ldots$

and using an "Expectation Maximisation" (EM) algorithm, the hyperparameters $\mu_{i}$ and $v_{i}$ can be estimated jointly, as shown in Appendix B (Friston et al., 2002). Having identified $\mathbf{C}_{\varepsilon}$ and $\mathbf{C}_{\mathbf{j}}$, j can be calculated easily with Eqs. (8a) and (8b).

With the 2-level approach, the unknown parameters $\mathbf{j}$ are assumed to be Gaussian variables with zero mean (shrinkage priors), as in Eq. (11). Regional variance can now be increased to render some locations more likely to be active. Indeed, a source with a larger variance is less constrained, thus more likely to be different from zero. Note that in Eqs. (15a) and (15b), we have a linear expansion of the covariance $\mathbf{C}_{\mathbf{j}}$ as opposed to the precision $\mathbf{C}_{\mathbf{j}}^{-1}$ (cf. Eq. (12)). Both formulations can be accommodated by EM. We will use Eqs. (15a) and (15b), noting that the prior covariance components $\mathbf{C}_{e_{2}, i}$ can be constructed to preclude unlikely sources.

Eq. (15b) allows a more accurate approximation of the source covariance matrix $\mathbf{C}_{\mathbf{j}}$ when its exact form is not known a priori. A variety of source constraints can be introduced simultaneously but, more importantly, the relative weight of each constraint is not fixed. This feature is particularly interesting when spatial coherence constraints and explicit location priors are used together. For example, a few sources can be specified as a priori more active: this can be done manually (from prior knowledge or past experience) or derived from haemodynamic measures of brain activity, such as thresholded statistical maps obtained from fMRI data. In this case, a 
set of activated regions can be identified. Because of the slowness of the haemodynamic response, these location priors carry no (or very little) temporal information (i.e., whether they have been simultaneously or successively active). With Eq. (15b), each location prior can be introduced separately with its own hyperparameter $v_{i}$.

Similarly, the noise covariance matrix $\mathbf{C}_{\varepsilon}$ can be modelled more accurately by Eq. (15a). For example, independent and uniform noise over the electrodes can be introduced by defining $\mathbf{C}_{e_{1}, 1}$ as the identity matrix. Covariance between close or distant electrodes can be introduced in $\mathbf{C}_{e_{1}, 2}$; this can generally be estimated from the data (e.g., within the pre-stimulus interval of the ERP). If a subset of electrodes picked up more noise than the others, this can also be modelled in $\mathbf{C}_{e_{1}, 3}$. The different noise covariance components are then balanced through the hyperparameters $\mu_{i}$.

By carefully selecting the EEG (or ERP) episode for which the hyperparameters are assumed stationary and the sources calculated, the EM algorithm should favour the relevant priors by increasing their hyperparameter and ignore the others by rendering their hyperparameters very small.

\section{Restricted maximum likelihood solution}

In practise, the system of Eqs. (13) (14a) (14b) (15a) (15b) is solved by minimising the "Restricted Maximum Likelihood" (ReML) objective function (see Appendix A.2). The two-level model of Eq. (13) can be collapsed into a single equation (see Appendix B.2):

$\mathbf{v}=\mathbf{L} \varepsilon_{2}+\varepsilon_{1}$

similar to Eq. (3). Then, from Eqs. (14a) and (14b), we can establish the relationship between the covariance matrices of the data, sources and noise:

$E\left\{\mathbf{v} \mathbf{v}^{t}\right\}=\mathbf{C}_{\mathbf{v}}=\mathbf{L} \mathbf{C}_{\mathbf{j}} \mathbf{L}^{t}+\mathbf{C}_{\varepsilon}$

where $\mathbf{C}_{\mathbf{v}}$ is the covariance matrix ${ }^{1}$ of the data $\mathbf{v}$.

With the linear expansion of the matrices $\mathbf{C}_{\varepsilon}$ and $\mathbf{C}_{\mathbf{j}}$, as defined in Eqs. (15a) and (15b), Eq. (17) becomes:

$\mathbf{C}_{\mathbf{v}}=\sum v_{i}\left(\mathbf{L} \mathbf{C}_{e_{2}, i} \mathbf{L}^{t}\right)+\sum \mu_{i} \mathbf{C}_{e_{1}, i}$

where only the hyperparameters $\mu_{i}$ and $v_{i}$ are unknown. The problem can be solved iteratively using the algorithm described in Appendix B.3. To get a very precise estimate of the hyperparameters $\mu_{i}$ and $v_{i}$, one can use multiple observations of EEG activity to calculate the covariance matrix of the data, $\mathbf{C}_{\mathbf{v}} \approx \mathbf{v v}^{t}$. This sample covariance can be based on successive time bins assuming that the noise and prior covariances are locally stationary. Although not pursued in this paper, it is also possible to use instantaneous estimates of $\mathbf{C}_{\mathbf{v}}$ sampled from the same time bin of multiple trials.

\section{Spatial constraints and priors}

Anatomical and physiological information derived from other imaging modalities are generally used to constrain the solution

\footnotetext{
${ }^{1}$ We refer to $\mathbf{C}_{\mathbf{v}}$ as a "covariance matrix" but, strictly speaking, it is just a second order matrix. Covariance matrices are second-order matrices of mean centered variables, whereas our variables were only baseline corrected (set to 0 ) at the beginning of the time series.
}

in the spatial domain. A two step approach was presented in Phillips et al. (2002a): First, the source distribution is modelled by a set of spatial basis function ("hard" constraints specified with infinite precision), then further "soft" constraints are introduced through $\mathbf{H}$ as in Eq. (9) for the classic WMN formulation, or through $\mathbf{C}_{e_{2}, i}$ as in Eq. (15b) for the hierarchical PEB approach. The sources priors, expressed as (co)variance matrices, are thus employed in two different ways: the anatomical priors are used to define spatial basis functions, while other priors enter in a probabilistic way. The orientation and location of the dipoles are embodied in the lead field matrix $\mathbf{L}$.

\section{Spatially informed basis functions}

The grey matter density at each dipole location can be represented by a diagonal matrix $\mathbf{G}\left(N_{d} \times N_{d}\right)$ encoding the prior variance in signal at different points in space. A convolution matrix D $\left(N_{d} \times N_{d}\right)$ is defined to impose spatial coherence among the dipoles. With these matrices $\mathbf{G}$ and $\mathbf{D}$ a set of "spatially Informed Basis Functions" (sIBF) B can be constructed from a prior covariance matrix $\mathbf{C}$, where

$\mathbf{C}=\mathbf{G}^{t / 2} \mathbf{D}^{t} \mathbf{D} \mathbf{G}^{1 / 2}$

Informed basis functions $\mathbf{B}$ are then obtained as the eigenvectors of $\mathbf{C}$ or, equivalently, by using the singular value decomposition of $\mathbf{C}^{1 / 2}=\mathbf{D G}^{1 / 2}$ :

$\mathbf{U S W}^{t}=\operatorname{svd}\left(\mathbf{D G}^{1 / 2}\right)$

Columns of $\mathbf{W}$ corresponding to normalised (by their mean) eigenvalues $\mathbf{S}^{2}$ greater than unity are retained to form the basis set B. Selecting these eigenvectors ensures a high mutual information between the signal before and after projection onto the basis set (under prior assumptions). In terms of the Bayesian formulation, this is equivalent to setting the prior variance of spatial modes (corresponding to the "minor" eigenvectors) to zero.

With $\mathbf{B}$ the source distribution $\mathbf{j}$ can be expressed as:

$\mathbf{B}^{t} \mathbf{j}=\mathbf{k} \Rightarrow \tilde{\mathbf{j}}=\mathbf{B} \mathbf{k}$

where $\mathbf{k}$ is a vector of size $N_{k} \times 1$ with $N_{k} \ll N_{d}$. In fact $\tilde{\mathbf{j}}$ is the source distribution restricted to the subspace spanned by the columns of $\mathbf{B}$, i.e., the sIBF. By considering $\tilde{\mathbf{j}}$ as a spatially constrained source distribution, and substituting Eq. (21) into Eqs. (10a), (10b) or (13), the unknowns become $\mathbf{k}$ and the size of the solution space is reduced from $N_{d}$ to $N_{k}$.

The linear problem (3) (and Eq. (4)) can be rewritten in terms of $\mathbf{k}$ (and $\mathbf{K}$ ) instead of $\mathbf{j}$ (and $\mathbf{J}$ ):

$\mathbf{v}=\mathbf{L}_{\mathbf{B}} \mathbf{k}+\varepsilon$

$\mathbf{V}=\mathbf{L}_{\mathbf{B}} \mathbf{K}+\varepsilon$

where $\mathbf{L}_{\mathbf{B}}=\mathbf{L B}$. With this change of variables from $\mathbf{j}$ to $\mathbf{k}$, the covariance matrix of the sources $\mathbf{C}_{\mathbf{j}}$ has to be projected by $\mathbf{B}$ to obtain the covariance matrix $\mathbf{C}_{\mathbf{k}}$ :

$\mathbf{C}_{\mathbf{k}}=\mathbf{B}^{t} \mathbf{C}_{\mathbf{j}} \mathbf{B}$

and Eq. (18) becomes

$\mathbf{C}_{\mathbf{v}}=\sum v_{i}\left(\mathbf{L}_{\mathbf{B}} \mathbf{C}_{\mathbf{B} e_{2}, i} \mathbf{L}_{\mathbf{B}}^{t}\right)+\sum \mu_{i} \mathbf{C}_{e_{1}, i}$

where $\mathbf{C}_{\mathbf{B} e_{2}, i}=\mathbf{B}^{t} \mathbf{C}_{e_{2}, i} \mathbf{B}$. 


\section{Soft spatial constraints}

The probabilistic constraints enter as the matrices $\mathbf{C}_{e_{2}, i}$ as in Eq. (15b). Here we will consider three types.

First, the major modes, retained to form the basis set $\mathbf{C}_{\mathbf{k}}$, are entered into Eq. (15b) by putting their associated eigenvalues on the main diagonal of $\mathbf{C}_{e_{2}}, 1$. These constraints imply that the basis function with the highest mode (i.e., with the lowest spatial frequencies) is more likely to represent the source activity, ensuring a smooth solution.

To ensure that sources are likely to influence the electrical potential equally at the electrodes irrespective of their depth (Grave de Peralta Menendez and Gonzalez Andino, 1998; Ioannides et al., 1990; Pascual-Marqui, 1999), deeper sources are given a larger a priori variance than superficial sources. The depth is indexed by the norm of the lead field for each source: the covariance component $\mathbf{C}_{e_{2}, 2}$ of $\mathbf{C}_{\mathbf{j}}$ is defined by the diagonal matrix $\left(\operatorname{diag}\left(\mathbf{L}^{t} \mathbf{L}\right)\right)^{-1}$.

Finally, location priors can be introduced on leading diagonal matrices $\mathbf{C}_{e_{2}, i}$ (where $i>2$ ) with elements that reflect the prior probability of whether the source is active or not. Here we only allow values of 0 , the variance is to be left unchanged, or 1 , the variance is increased according to the value of the corresponding hyperparameter $v_{i}$.

\section{Applications}

In this section, the solution introduced in the previous section is assessed. We first use a simplified model with synthetic data to evaluate the solution proposed in comparison with more "classic" solutions. Afterwards, the method is applied to a real data set: ERP data recorded from a normal subject and a realistic head model based on a structural MRI scan.

The solution combining the ReML estimation of the hyperparameters and constraining the solution space with "spatially Informed Basis Functions" (sIBF) will be denoted "EM-IBF solution".

\section{Comparison methods}

The EM-IBF approach was compared with two other commonly employed approaches: a Weighted Minimum Norm (WMN) solution and a Maximum Smoothness (MS) solution. For both cases, we use an EM scheme to estimate the hyperparameters; therefore, they will be named "EM-WMN" and "EM-MS".

The EM-WMN approach is similar to the EM-IBF solution, except that the solution space was not constrained a priori by the sIBF. The solution is obtained with Eqs. (8a) and (8b), after the noise and source covariance matrices have been estimated with the ReML procedure. The depth weighting constraints and the location priors were the same for EM-IBF and EM-WMN, but the spatial coherence constraint was defined with a convolution matrix, the same one used to calculate the SIBF (see "Spatially informed basis functions" section) for EM-WMN.

The MS solution is simply a particular case of Eqs. (5a) and (5b), (7a) and (7b). The solution space is not constrained a priori by the sIBF and the weighting matrix $\mathbf{H}$ is defined as a weighted three-dimensional Laplacian, $\mathbf{H}=\mathbf{M W}$. $\mathbf{W}$ is a leading diagonal matrix defined by $\left(\operatorname{diag}\left(\mathbf{L}^{t} \mathbf{L}\right)\right)^{1 / 2}$, which is the inverse of the square root of the depth weighting used for the EM-IBF and EM-WMN solutions. The Laplacian matrix $\mathbf{M}$ is a regularised discrete three- dimensional second-order derivative operator defined as in Pascual-Marqui (1999). Thus, $\mathbf{C}_{\mathbf{j}}$ is modelled by a single term $\mathbf{C}_{e_{2}, 1}=\left(\mathbf{H}^{t} \mathbf{H}\right)^{-1}=\left(\mathbf{W}^{t} \mathbf{M}^{t} \mathbf{M W}\right)^{-1}$, and only one $v_{i}$ is estimated with EM-MS. In the "classic" implementation of the MS solution, there is no means to include priors other than the maximum smoothness constraint; therefore, no location priors were used in this assessment. In the literature (Pascual-Marqui, 1999; PascualMarqui et al., 1994), the MS solution is usually implemented in the LORETA software (Pascual-Marqui, 1998) for the ideal noise-free case, using Eqs. (7a) and (7b) with $\lambda \rightarrow 0$ (although PascualMarqui, 1995 has suggested employing noise regularisation by taking $\lambda \neq 0$ ). We reproduced this $\lambda=0$ analysis in our simulations and refer to it as "MS with no regularisation".

The main objective of these simulations was to establish the face and construct validity of the ReML hyperparameter estimates. We hoped to show that the ReML estimators were sensitive to different levels of noise variance and behaved adaptively and appropriately. Furthermore, we wanted to establish this face validity using a series of qualitatively different priors.

\section{Simulations with a simplified model}

For a thorough comparison of the various methods introduced in this paper, we use a simplified head model with synthetic data. With this model and data, we are able to simulate different level of noise as well as different types of locations priors (accurate vs. inaccurate).

\section{Simplified model and synthetic data}

The source model consisted of 1716 dipoles distributed uniformly on an horizontal grid (with a maximum of 24 sources along a radius), within a three-sphere shell model. Twenty-seven electrodes were placed on the upper hemisphere according to a pseudo 10-20 electrode setup; see Fig. 1. The orientations of each source were fixed and the lead-field $\mathbf{L}$ for all the sources calculated analytically (Ary et al., 1981). The density of grey matter was assumed to vary over the dipole location as shown in Fig. 1.

In the simulations presented below, the spatial coherence between the sources was modelled by the matrix D (from "Spatially informed basis functions" section) by using a Gaussian function, with $\sigma=2 \times$ "grid size". With this spatial coherence, the solution space was reduced from 1716 independent sources to 207 orthogonal sIBF.

A set of 200 locations were selected randomly to assess the efficiency of the methods presented in "Hierarchical parametric empirical Bayes approach" and "Comparison methods" sections. At each of these locations, an instantaneous distributed source set $\mathbf{j}_{o}$ was generated as a set of connected dipoles within a 1.5 grid-size radius of a "central" dipole. On average, each source comprises 8.7 dipoles; see Fig. 2. Simulated location priors centered as a disk of 4 grid-size radius, i.e., on average 45 sources. Selected sources were defined as being a priori active. In the simulations presented below, we used three kinds of location priors: (1) accurate priors, centred on the active source set, (2) close, inaccurate priors, located between 6 and 16 grid-size from the truly activated source, and (3) distant, inaccurate priors, between 24 and 48 grid-size from the truly activated source; see Fig. 2. The prior covariance components on the sources $\mathbf{C}_{e_{2}, i}$ were normalised such that the Frobenius norm of each matrix, projected onto the subspace spanned by the sIBF, was unity. 

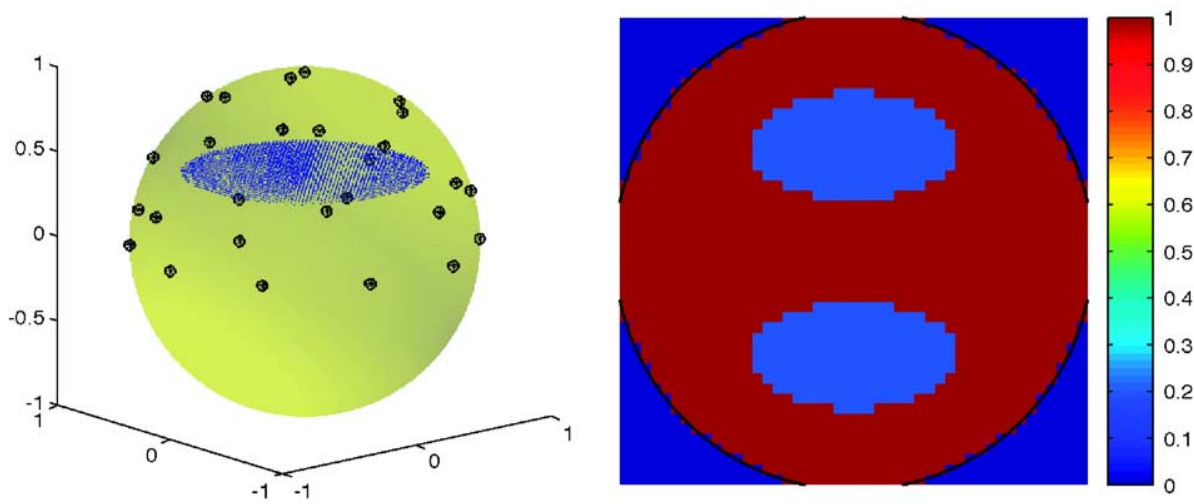

Fig. 1. Simplified head model: 1716 dipoles within a three-sphere shell model with 27 electrodes (left), and "grey matter" density over the source locations (right).

Each source vector $\mathbf{j}_{o}$ was modulated over time to generate a time series $\mathbf{J}_{o}$. The time course of the resulting EEG is shown in Fig. 3. Data with different signal-to-noise-ratio (SNR) were obtained by adding scaled white noise $\varepsilon$ to the noise free data $\mathbf{V}=$ $\mathbf{L J}_{o}+\varepsilon$. Three levels of noise were used by adopting a SNR of 4, 12 and 100. The SNR is defined here as the ratio between the norm of the signal (at its maximum) and the norm of the added noise component. With an SNR at 100, data are almost noise free, still the noise variance is not zero and should be estimable. The electrical potential at the electrodes, over time, with these three SNRs is shown in Fig. 3.

\section{Assessment criteria}

Two criteria were used to compare the performance of the different methods (EM-IBF, EM-WMN and EM-MS): "Localisation Error" (LE) and "Root Mean Square Error" (RMSE) (see also Phillips et al., 2002a,b). The LE is defined as the distance between the location $\vec{r}_{r}$ of the maximum (absolute value) of the

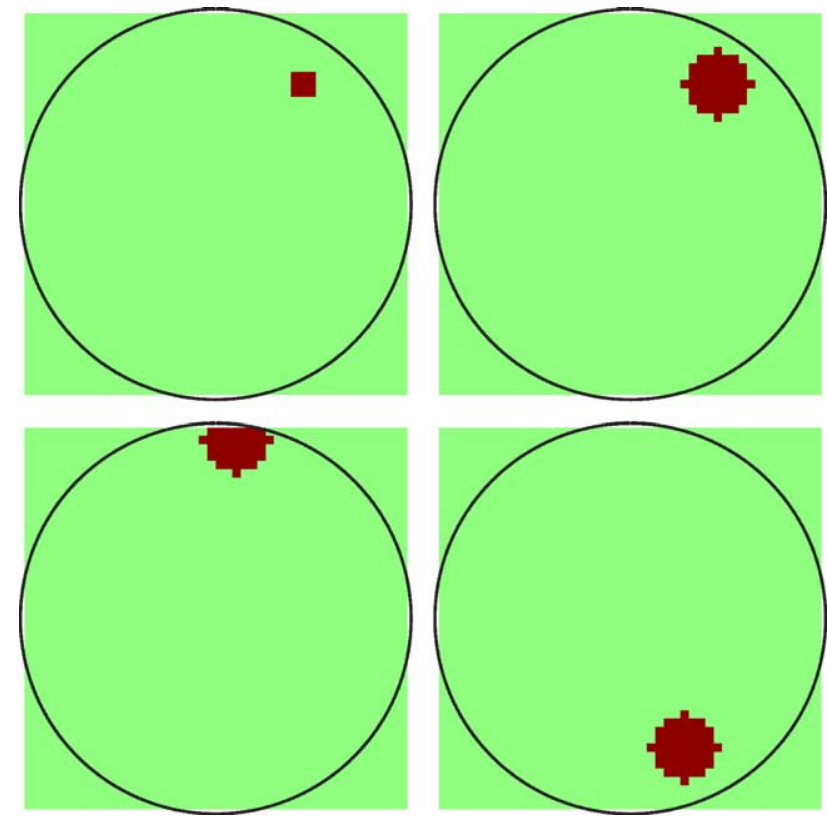

Fig. 2. Example of a source used in the simulations (top left) with the corresponding accurate location priors (top right), as well as inaccurate location priors (close, bottom left, and distant, bottom right). reconstructed source amplitudes $\mathbf{j}_{r}$ and the location $\vec{r}_{o}$ of the original source set $\mathbf{j}_{o}$. To obtain a single value of LE for the estimated and actual distributed source $\left(\mathbf{J}_{o}, \mathbf{J}_{r}\right)$ over time, the following procedure was adopted: nine time points around the maximum of the original data were considered. For this subset of reconstructions $\mathbf{j}_{r, i}$, the $\mathrm{LE}_{i}$ was calculated. The largest value of $\mathrm{LE}_{i}$ was retained as the "worst case LE" for $\left(\mathbf{J}_{o}, \mathbf{J}_{r}\right)$.

The RMSE is defined as the Frobenius norm of the difference of the scaled distributions:

$\mathrm{RMSE}=\left\|\mathbf{J}_{r}^{*}-\mathbf{J}_{o}^{*}\right\|_{F r o}$

where $\mathbf{J}_{r}^{*}=\mathbf{J}_{r} / j_{r, \text { max }}$ and $\mathbf{J}_{o}^{*}=\mathbf{J}_{o} / j_{o, \text { max }}$ where $j_{\text {o,max }}$ represents the amplitude of the largest (absolute value) dipole in $\mathbf{J}_{o}$. This ensures that the Root Mean Square Error measures the discrepancy between the original and reconstructed source distributions without any global scaling effect.

The "Localisation Error" (LE) provides a measure of the localisation accuracy of the reconstruction method, a small value of LE indicates that the location of the original source was recovered well. The "Root Mean Square Error" (RMSE) measures the "goodness of fit" of the reconstruction. A small value of RMSE indicates a small discrepancy between the original and reconstructed source distributions. The RMSE is only useful to further compare two solutions that have approximately the same LE. If both solutions have almost the same LE, the one with the smaller RMSE would be preferred as the reconstructed source is then more focal. A very focal reconstructed source with a large LE may have a smaller RMSE than a blurred reconstructed source with a small LE, but the latter solution, although over-smoothed, provides at least some location information.

Each simulation condition (SNR, location prior) was assessed for 200 different source configurations, a "LE bound" (maxLE) was calculated from the LE of all the 200 reconstructions, such that $80 \%$ of the sources were recovered within this bound. The RMSE from the 200 sources was summarised by its mean.

\section{Simulations}

In this subsection, we present the results of the reconstructions for the three SNRs and for the four different location priors: without any location priors, with accurate location priors, with inaccurate location priors, and with both accurate and inaccurate location priors. Figs. 4-6 show exemplar reconstructions using the EM-IBF, EM-WMN and EM-MS solutions, respectively. The source set and location priors used correspond to those shown in 

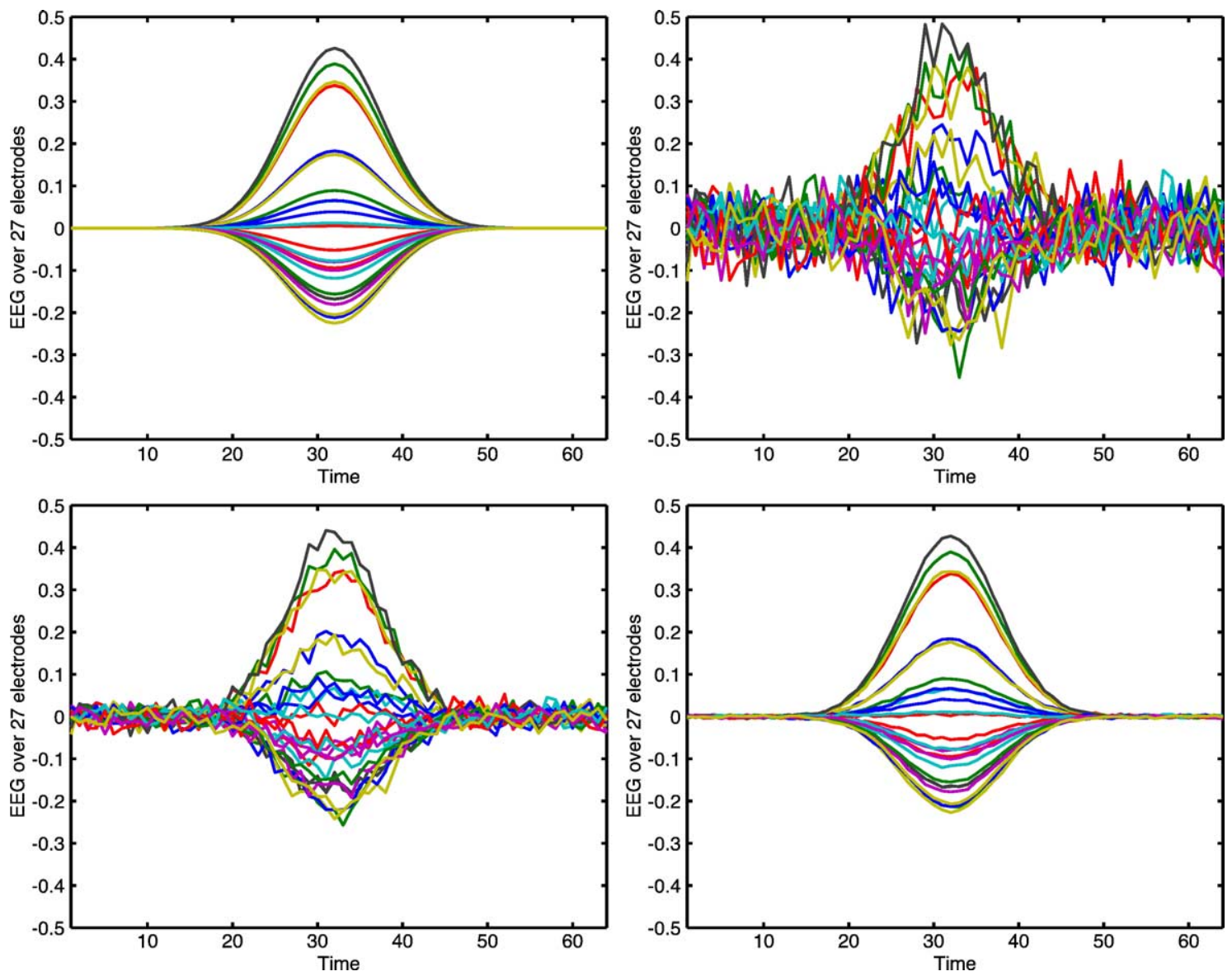

Fig. 3. Synthetic data: original data without noise (top left). Same data with added noise at different SNRs: 4 (top right), 12 (bottom left), 100 (bottom right).
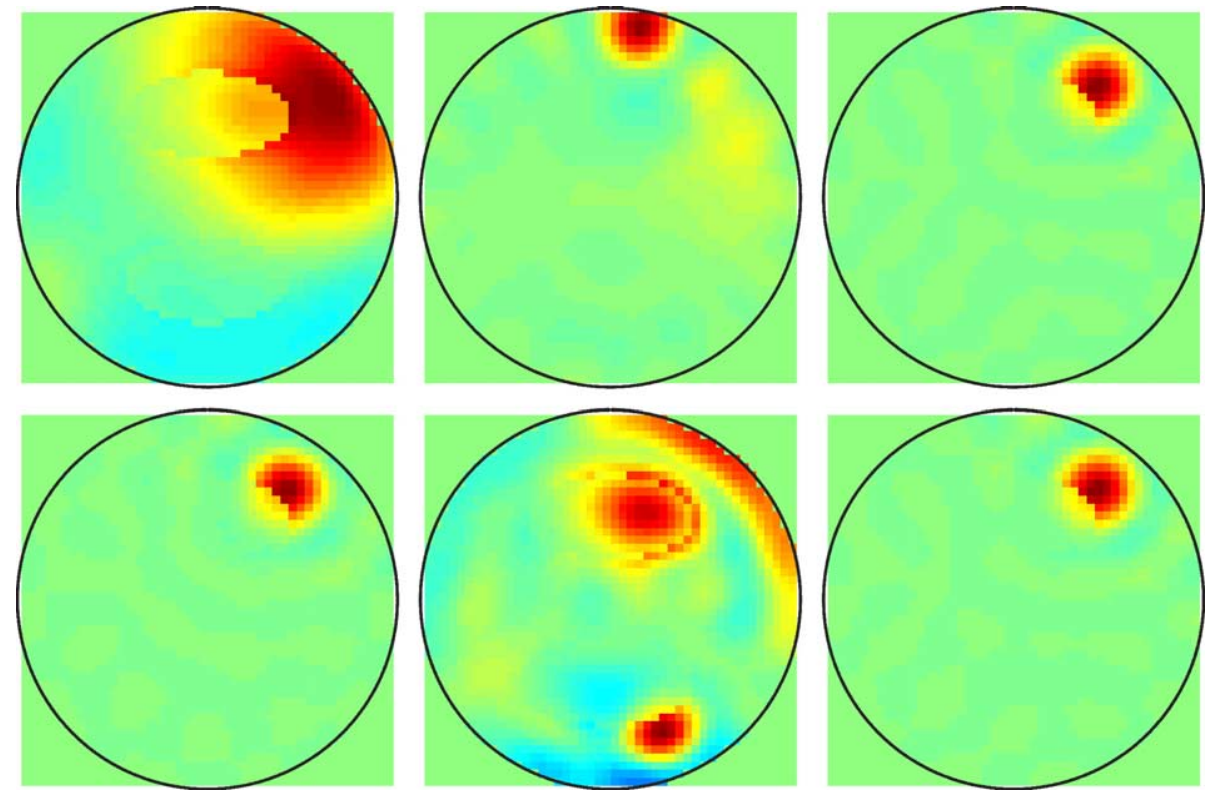

Fig. 4. Reconstructed sources with the EM-IBF method ( $\mathrm{SNR}=12$ ): no location priors (top left), with accurate location priors (bottom left), with inaccurate close priors (top middle), with inaccurate distant priors (bottom middle), with both accurate and close inaccurate priors (top right), and with both accurate and distant inaccurate priors (bottom right). 

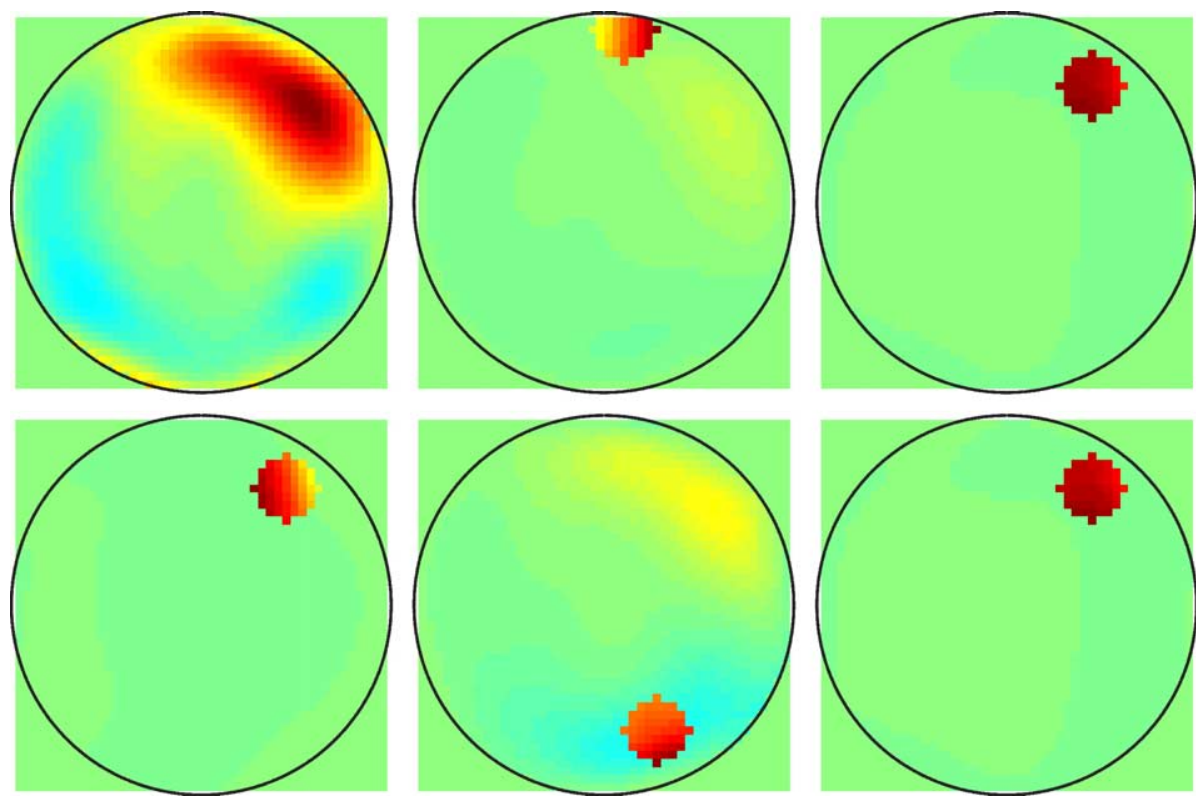

Fig. 5. Reconstructed sources with the EM-WMN method ( $\mathrm{SNR}=12$ ): no location priors (top left), with accurate location priors (bottom left), with inaccurate close priors (top middle), with inaccurate distant priors (bottom middle), with both accurate and close inaccurate priors (top right), and with both accurate and distant inaccurate priors (bottom right).

Fig. 2. The solutions were applied to the EEG data shown in Fig. 3, with the intermediate SNR (SNR = 12).

\section{LE and RMSE}

Tables 1 and 2 summarise the $80 \%$ LE bound and mean RMSE for the three solutions (EM-IBF, EM-WMN and EM-MS), the three SNRs and the four location priors.

It is clear that, in general, the EM-IBF solution is better than the EM-WMN. The EM-MS gives slightly better results than the EMIBF solution without accurate location priors but, if the data are assumed to be noise-free, i.e., without the noise component in Eq. (17), the MS solution simply does not work.

The introduction of accurate priors dramatically reduces the LE and RMSE for both EM-IBF and EM-WMN but the EM-WMN solution is still outperformed by the EM-IBF solution. If only inaccurate priors are introduced, then the solutions are inappropriately biased towards those inaccurate priors, giving meaningless results. On the contrary, the combination of accurate and inaccurate location priors leads to a precise reconstruction, as the inaccurate location priors are effectively discarded in the ReML procedure. The performance of all methods increases with the SNR.
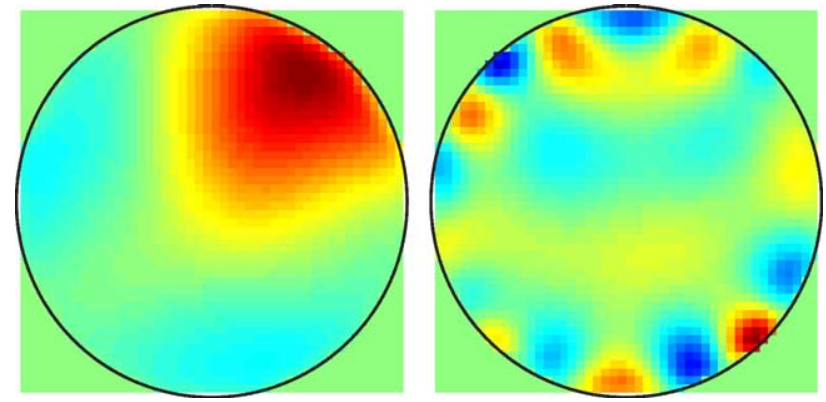

Fig. 6. Reconstructed sources with the MS method $(\mathrm{SNR}=12)$ : with EM regularisation (left), assuming the data are noise-free (right).

\section{Hyperparameters estimates}

The estimation of the first hyperparameter representing the estimated noise variance [corresponding to $\mu_{1}$ in Eqs. (15a) and (15b)] was relatively stable throughout all the simulations, as seen in Table 3. These estimated values reflected accurately the actual variance of white noise added for the simulations, i.e., 2.3 (low SNR), 0.26 (medium SNR) and 0.0037 (high SNR). Note that it is difficult to interpret the value of the hyperparameters as their "absolute" values depends on the nature and scaling of the covariance components, sIBF and lead field matrix. Therefore, only their relative values are useful.

Although the estimated noise variance is, on average, accurate, the standard deviation of its estimate is much smaller (at least one order) when accurate location priors are used. The inclusion of accurate location priors seems to help the partioning of signal variance into its components, namely source activity and additive noise. For some sources, the noise variance was greatly overestimated.

Tables 4 and 5 summarise the mean value and standard deviation of the hyperparameters relative to the source covariance for the EM-IBF and EM-WMN solution. The values of the hyperparameter relative to the source variance in the EM-MS method are $0.52 \pm 0.065,0.56 \pm 0.26$ and $0.6 \pm 0.46$ for the low, medium and high SNR, respectively.

Some hyperparameters are negative. This simply means that the corresponding covariance component was used to reduce the (co-) variance of the sources. The combination of all the covariance components should always lead to a positive definite (co-)variance matrix estimate. In general, the hyperparameter means decrease as the SNR increases, except when inaccurate location priors are used in isolation. The use of accurate location priors has a large influence on the other priors: Indeed, the hyperparameter corresponding to the accurate location priors is several orders larger than any other hyperparameter (corresponding to the spatial coherence, depth or inaccurate location priors). 
Table 1

Localisation error (maxLE) for the three methods, the three SNRs and the four location priors used (none, accurate, inaccurate, and both accurate and inaccurate)

\begin{tabular}{|c|c|c|c|c|c|c|c|c|c|}
\hline \multirow[t]{2}{*}{ SNR } & \multicolumn{3}{|c|}{ EM-IBF } & \multicolumn{3}{|c|}{ EM-WMN } & \multicolumn{3}{|c|}{ EM-MS } \\
\hline & Low & Medium & High & Low & Medium & High & Low & Medium & High \\
\hline \multirow[t]{2}{*}{ No priors } & 7 & 5 & 3 & 6 & 4 & 10 & 4 & 3 & 2 \\
\hline & & & & & & & 42 & 42 & 29 \\
\hline Accurate priors & 2 & 2 & 1 & 4 & 4 & 4 & - & - & - \\
\hline \multirow[t]{2}{*}{ Inaccurate priors } & 13 & 12 & 10 & 12 & 15 & 13 & - & - & - \\
\hline & 31 & 28 & 28 & 34 & 32 & 32 & & & \\
\hline \multirow[t]{2}{*}{ Accurate and inaccurate priors } & 2 & 2 & 1 & 4 & 4 & 4 & - & - & - \\
\hline & 2 & 2 & 1 & 4 & 4 & 4 & & & \\
\hline
\end{tabular}

The LE is expressed as the maximum LE required to recover at least $80 \%$ of the sources within this bound. Units are in grid element size. The second line of maxLE for the MS solution corresponds to the case where no noise regularisation was applied, i.e., the data were considered noise-free. When inaccurate priors are used, the first (resp. second) line of maxLE corresponds to close (resp. distant) inaccurate priors.

Apart from the hyperparameter corresponding to the accurate location priors, all the other hyperparameters vary over a relatively large range and can have positive or negative values depending on the source, the noise and priors used. Nevertheless, with some exceptions, the standard deviation of the hyperparameter estimates decreases as the SNR increases.

\section{Real source reconstruction}

The method described in the previous sections was applied to a set of somatosensory-evoked response data. EEG data were acquired from a healthy subject (male, right-handed). Fifty-nine electrodes were placed on the scalp according to the extended 1020 system and recorded with respect to a cephalic reference. All channels were re-referenced to an average cephalic reference. Extra channels were used to record the electro-occulogram (horizontal and vertical), allowing trials contaminated by EOG artefact to be rejected. One thousand five hundred electrical stimulations were applied to the right median nerve. The stimulus onset asynchrony (SOA) was selected randomly (uniform distribution) between 400 and $500 \mathrm{~ms}$. The sampling rate was $1000 \mathrm{~Hz}$ and the data were filtered between 0.05 and $40 \mathrm{~Hz}$. The continuous EEG data were epoched from $100 \mathrm{~ms}$ pre-stimulus to $156 \mathrm{~ms}$ afterwards. Epochs exhibiting EOG or other artefact were rejected manually. ERP data were obtained by averaging the remaining epochs; see Fig. 7.

Electrode locations were recorded using a three-dimensional digitiser (Polhemus Fastrak, 2003) and the structural MRI (sMRI) of the subject was used to build the head and source model. The grey-white matter interface was extracted from the sMRI with the "Anatomist" software (Rivière and Papadopoulos-Orfanos, 2003;
Rivière et al., 2000) and 15,991 sources were uniformally distributed on this surface. The forward problem was then solved using an approach described by Spinelli et al. (2000): The head and source model were mapped to a spherical system (three sphere shell model) by defining warping operations that transformed the head model to a best-fitting sphere. The forward solution was then calculated for each dipole using an analytical solution (Cuffin and Cohen, 1979). Spatially informed basis functions (sIBF) were calculated as described in "Spatially informed basis function": the prior covariance matrix $\mathbf{G}$ was the identity matrix and the convolution matrix D was based on a Gaussian function (with a kernel of $10 \mathrm{~mm}$, effectively defining the minimum radius of an activated cortical patch) of the distance between dipoles on the cortical surface. The 15,991 independent sources were modelled by 669 sIBF. To account for possible error in source orientation, each source comprised one dipole perpendicular to the surface, plus two orthogonal dipoles oriented tangentially to the local surface. This is equivalent to the case where source orientation is left free (see "Theory" section): $\mathbf{j}=\left[\overrightarrow{j_{1}}{\overrightarrow{j_{2}}}^{t} \ldots{\overrightarrow{j_{N}}}_{N_{d}}^{t}\right]^{t}$, where $\overrightarrow{j_{i}}=\left[j_{x, i} j_{y, i} j_{z, i}\right]^{t}$ but here each source $\overrightarrow{j_{i}} i$ is rotated such that $\overrightarrow{j_{i}}=\left[j_{\perp, i} j_{/ / 1, i} j_{/ / 2, i}\right]^{t}$.

For the source reconstruction, we focused on the N20 wave because its source has been previously shown to be located in the primary sensory cortex (Desmedt and Cheron, 1981). We used a time window extending from 20 to $25 \mathrm{~ms}$ around the N20 peak (at $23 \mathrm{~ms}$ ). Fig. 8 shows the N20 potential distribution over the actual scalp surface of the volunteer, as estimated with a spherical spline interpolation (Perrin et al., 1989).

For the reconstruction, the noise was modelled by two covariance components: $\mathbf{C}_{e_{1}, 1}$ was an estimate of the noise covariance based on the averaged pre-stimulus ERP signal

Table 2

Mean RMSE for the three methods, the three SNRs and the four location priors used (none, accurate, inaccurate, and both accurate and inaccurate)

\begin{tabular}{|c|c|c|c|c|c|c|c|c|c|}
\hline \multirow[t]{2}{*}{ SNR } & \multicolumn{3}{|c|}{ EM-IBF } & \multicolumn{3}{|c|}{ EM-WMN } & \multicolumn{3}{|c|}{ EM-MS } \\
\hline & Low & Medium & High & Low & Medium & High & Low & Medium & High \\
\hline \multirow[t]{2}{*}{ No priors } & 35.6 & 29.5 & 24.1 & 40.3 & 33.9 & 29.3 & 50.0 & 39.1 & 29.5 \\
\hline & & & & & & & 35.5 & 35.4 & 35.0 \\
\hline Accurate priors & 12.4 & 11.7 & 9.2 & 14.0 & 13.7 & 11.6 & - & - & - \\
\hline \multirow[t]{2}{*}{ Inaccurate priors } & 24.0 & 22.9 & 19.9 & 24.5 & 23.5 & 21.1 & - & - & - \\
\hline & 29.3 & 28.1 & 24.9 & 33.0 & 31.6 & 27.7 & & & \\
\hline \multirow[t]{2}{*}{ Accurate and inaccurate priors } & 12.6 & 11.8 & 9.3 & 13.9 & 13.2 & 11.7 & - & - & - \\
\hline & 12.4 & 11.7 & 9.3 & 14.0 & 13.6 & 11.6 & & & \\
\hline
\end{tabular}

The second line of maxLE for the MS solution corresponds to the case where no noise regularisation was applied, i.e., the data were considered noise-free. When inaccurate priors are used, the first (resp. second) line of maxLE corresponds to close (resp. distant) inaccurate priors. 
Table 3

Mean value and standard deviation of the hyperparameters relative to the noise component

\begin{tabular}{|c|c|c|c|c|c|c|}
\hline \multirow[t]{2}{*}{ SNR } & \multicolumn{3}{|c|}{ Without accurate location priors } & \multicolumn{3}{|c|}{ With accurate location priors } \\
\hline & Low & Medium & High & Low & Medium & High \\
\hline EM-IBF & $2.3 \pm 0.14$ & $0.26 \pm 0.038$ & $0.0059 \pm 0.018$ & $2.3 \pm 0.04$ & $0.26 \pm 0.0065$ & $0.0037 \pm 0.00011$ \\
\hline EM-WMN & $2.3 \pm 0.15$ & $0.26 \pm 0.038$ & $0.0082 \pm 0.047$ & $2.3 \pm 0.04$ & $0.26 \pm 0.0065$ & $0.0037 \pm 0.00012$ \\
\hline EM-MS & $2.3 \pm 0.14$ & $0.26 \pm 0.026$ & $0.0042 \pm 0.0032$ & - & - & - \\
\hline
\end{tabular}

The values obtained were similar to the actual variance of the added noise component, i.e., 2.3 (low SNR), 0.26 (medium SNR) and 0.0037 (high SNR). The precision of this estimate depended mainly on the presence or absence of accurate location priors. Therefore, the simulations without any location priors and with inaccurate location priors are collapsed together in the category "solutions without accurate location priors". Similarly, results obtained "with accurate location priors" consider the two simulations with accurate location priors, with and without inaccurate location priors.

(effectively the covariance of the pre-stimulus ERP data), and $\mathbf{C}_{e_{1}, 2}$ was an identity matrix. For the source covariance constraints $\mathbf{C}_{e_{2}, i}$, we used the same constraints as in "Soft spatial constaints" section, namely spatial coherence and depth constraints. The $\mathbf{C}_{e_{1}, i}$ and $\mathbf{C}_{e_{2}, i}$, projected on the sIBF subspace, were normalised to unit Frobenius norm. To constrain the reconstructed sources to be approximately orthogonal to the cortical surface, the variance of the tangential dipoles was reduced by a factor of 0.2588 [corresponding to $\sin \left(15^{\circ}\right)$ ] relative to the dipole orthogonal to the cortical surface. This means the source orientation is allowed to vary slightly around the estimated optimal direction, i.e., perpendicular to the extracted cortical surface.

The reconstruction was first performed without any other location priors. Fig. 9 shows the norm of the reconstructed activity at each location, thresholded at $2 / 3$ of its maximum. As expected, activity is found in the left hemisphere, contralateral to the stimulus. The maximum of activity was found on the posterior bank of the central sulcus but there is also some apparently spurious reconstructed activity in the occipital lobe. The value of the hyperparameters corresponding to the two noise components (prestimulus covariance and identity matrices) were 9.738 and 0.104 , respectively. The values of the source covariance components (spatial coherence and depth) were 107.1 and 99.76, respectively.

A location prior was then introduced to further constrain the reconstruction. The region around the central sulcus, in both hemispheres, was selected and extracted with the electronic stereotactic brain atlas "WFU Pickatlas" (Maldjian et al., 2003) (see Fig. 10). The diagonal elements of the third prior covariance constraint, corresponding to dipoles within the highlighted area were set to 1 , and the others to $10^{-6}$. The a priori variance of these selected sources could thus be increased through the corresponding hyperparameter $\left[v_{3}\right.$ in Eq. (24)]. Note that this constraint will only be used if the data support it and give a suitably large ReML estimate of $v_{3}$.

With this extra source constraint, the reconstructed activity showed a single maximum on the posterior side of the central gyrus. Fig. 11 shows the norm of the reconstructed activity at each location, thresholded at $2 / 3$ of its maximum. The source with maximal amplitude is represented by the yellow arrow highlighting its location and orientation. The activity of the source with maximum norm is mostly determined by its orthogonal dipole. The two tangential dipoles are still useful as they allow the source to deviate by $1.45^{\circ}$ from the orthogonality to the cortical surface. The value of the hyperparameter corresponding to the location prior was 46.74 and the other hyperparameters were approximately the same as without location priors.

\section{Discussion}

The hierarchical PEB and ReML (EM) solutions presented here seem a good approach to EEG source localisation. The simulation with synthetic data and a simplified model showed that:

- In all cases, the noise variance estimate was accurate on average;

- The localisation error (LE) and Root Mean Square error (RMSE) were greatly reduced by the introduction of an accurate location prior.

- Inaccurate location priors could mislead the solution but if accurate and inaccurate location priors were used simultane-

Table 4

Mean value and standard deviation of the hyperparameters pertaining to the source variance for the EM-IBF solution

\begin{tabular}{|c|c|c|c|c|c|}
\hline & SNR & Spatial coherence, $S^{2}$ & Depth constraint & Accurate location & Inaccurate location \\
\hline \multirow[t]{3}{*}{ No priors } & Low & $-0.65 \pm 1.6$ & $2.5 \pm 3.7$ & - & - \\
\hline & Medium & $-0.43 \pm 1.3$ & $1.9 \pm 3.0$ & - & - \\
\hline & High & $-0.16 \pm 1.1$ & $1.2 \pm 2.2$ & - & - \\
\hline \multirow[t]{3}{*}{ Accurate priors } & Low & $0.015 \pm 0.038$ & $-0.058 \pm 0.087$ & $4.5 \pm 1.1$ & - \\
\hline & Medium & $0.0042 \pm 0.0068$ & $-0.015 \pm 0.017$ & $2.6 \pm 0.59$ & - \\
\hline & High & $0.00014 \pm 0.00026$ & $-.00054 \pm 0.00073$ & $1.8 \pm 0.61$ & - \\
\hline \multirow[t]{3}{*}{ Inaccurate priors } & Low & $-0.57 \pm 1.5$ & $2.2 \pm 3.5$ & - & $1.2 \pm 4.7$ \\
\hline & Medium & $-0.41 \pm 1.2$ & $1.7 \pm 2.7$ & - & $4.6 \pm 16$ \\
\hline & High & $-0.17 \pm 0.96$ & $1.1 \pm 2.0$ & - & $13 \pm 41$ \\
\hline \multirow[t]{3}{*}{ Accurate and inaccurate priors } & Low & $0.016 \pm 0.045$ & $-0.06 \pm 0.11$ & $4.6 \pm 1.1$ & $-0.037 \pm 0.093$ \\
\hline & Medium & $0.0046 \pm 0.0096$ & $-0.016 \pm 0.025$ & $2.7 \pm 0.61$ & $-0.02 \pm 0.038$ \\
\hline & High & $0.00014 \pm 0.00033$ & $-0.00054 \pm 0.00095$ & $1.8 \pm 0.59$ & $-0.00061 \pm 0.0031$ \\
\hline
\end{tabular}

The values of the single hyperparameters in the EM-MS method are $0.52 \pm 0.065,0.56 \pm 0.26$ and $0.6 \pm 0.46$ for the low, medium and high SNR, respectively. 
Table 5

Mean value and standard deviation of the hyperparameters pertaining to the source variance for the EM-WMN solution

\begin{tabular}{|c|c|c|c|c|c|}
\hline & SNR & Spatial coherence & Depth constraint & Accurate location & Inaccurate location \\
\hline \multirow[t]{3}{*}{ No priors } & Low & $-0.5 \pm 1.9$ & $6.8 \pm 14.0$ & - & - \\
\hline & Medium & $-0.35 \pm 1.7$ & $5.6 \pm 12.0$ & - & - \\
\hline & High & $-0.058 \pm 1.3$ & $3.1 \pm 8.8$ & - & - \\
\hline \multirow[t]{3}{*}{ Accurate priors } & Low & $0.016 \pm 0.043$ & $-0.2 \pm 0.32$ & $16 \pm 4.1$ & - \\
\hline & Medium & $0.0073 \pm 0.0097$ & $-.072 \pm 0.076$ & $9.1 \pm 1.9$ & - \\
\hline & High & $0.00016 \pm 0.00032$ & $-0.0016 \pm 0.0026$ & $6.9 \pm 1.6$ & - \\
\hline \multirow[t]{3}{*}{ Inaccurate priors } & Low & $-0.39 \pm 1.7$ & $5.6 \pm 13$ & - & $4.7 \pm 19$ \\
\hline & Medium & $-0.41 \pm 1.5$ & $5.5 \pm 11$ & - & $18 \pm 72$ \\
\hline & High & $-0.093 \pm 1.2$ & $2.9 \pm 7.9$ & - & $70 \pm 300$ \\
\hline \multirow[t]{3}{*}{ Accurate and inaccurate priors } & Low & $0.021 \pm 0.064$ & $-0.23 \pm 0.49$ & $16 \pm 4.4$ & $-0.15 \pm 0.36$ \\
\hline & Medium & $0.0088 \pm 0.014$ & $-0.082 \pm 0.11$ & $9.4 \pm 2.1$ & $-0.076 \pm 0.14$ \\
\hline & High & $0.00017 \pm 0.00034$ & $-0.0016 \pm 0.0029$ & $6.9 \pm 1.6$ & $-0.0021 \pm 0.013$ \\
\hline
\end{tabular}

The values of the single hyperparameters in the EM-MS method are $0.52 \pm 0.065,0.56 \pm 0.26$ and $0.6 \pm 0.46$ for the low, medium and high SNR, respectively.

ously, the inaccurate location prior had no effect on the source reconstruction.

The results obtained in the previous section show that the method can be used with real data. The orientation constraint is also very important. Indeed, with a realistic head model and the extracted cortical surface, the location and orientation of the sources could be fixed with respect to the estimated surface. Error committed on the location of a source will not have much influence on the solution of the inverse problem: the lead fields of two parallel dipoles, placed close together, are very similar (because of the physical spatial blurring of the signal on the scalp). On the contrary, error on the orientation of the source, i.e., the local curvature of the estimated cortical surface, can have a substantial impact on the inverse problem: the leadfields of two dipoles at the same location but with different orientations can be very different (orientation-free sources are actually modelled by three orthogonal dipoles at the same location).

In our case, if the sources were constrained to be perfectly orthogonal to the extracted cortical surface, the source reconstruction without the location prior was less accurate and presented more spurious sources in the parietal and occipital lobe (results not shown). By modelling each source with three orthogonal dipoles and

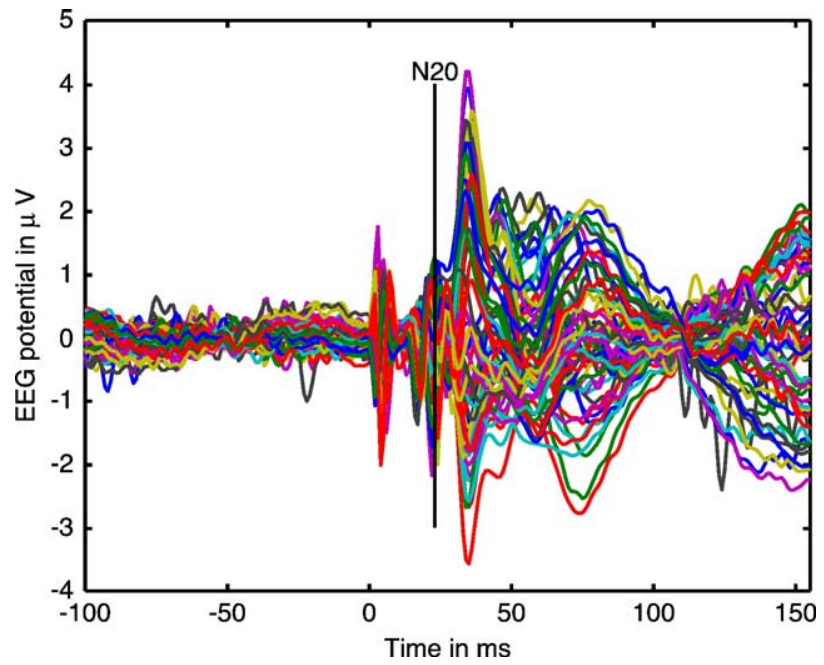

Fig. 7. Somatosensory ERP data from the 59 channels. At time $0 \mathrm{~ms}$, the artefact generated by the stimulator is clearly visible. The N20 wave reaches its maximum $23 \mathrm{~ms}$ after the stimulus. constraining the variance of the two tangential dipoles relative to the perpendicular one (with respect to the cortical surface), the source is constrained to be almost orthogonal to the cortical surface but has freedom to accommodate errors on the curvature of the cortical surface.

\section{Conclusions}

Combining data obtained from different techniques within the same mathematical framework is a way to overcome the intrinsic limitations (on temporal or spatial resolution) of individual brain imaging modalities. Such integration should provide an optimal solution that harnesses the strengths of each technique. In this paper, we have outlined a way in which structural and functional data can be used as priors in the estimation of EEG sources. Crucially, we have illustrated the role of ReML hyperparameter estimates in modelling the relative contributions of EEG residuals and MRI-based priors to the estimation.

The approach adopted in this work does not follow strictly the scheme of a distributed linear reconstruction. The spatial IBF, obtained from the anatomical information, allow constraints on the source localisation to enter in two separable steps. First, the dimensionality of the problem is reduced by projecting the solution

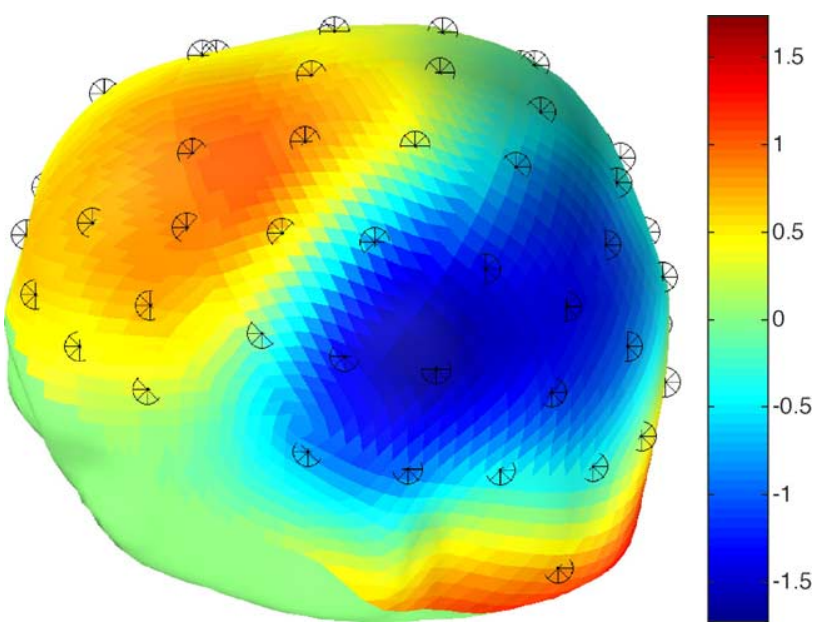

Fig. 8. N20 potential distribution over the over the scalp surface of the volunteer. Electrode locations are shown with the black markers 


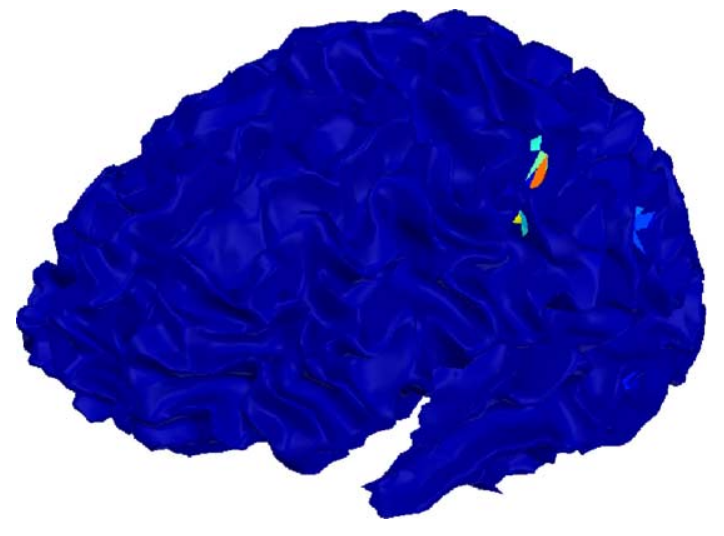

Fig. 9. Source reconstruction (norm of the sources thresholded at $2 / 3$ of its maximum and normalised between 0 and 1) without any prior location constraints.

space onto a subspace spanned by the spatial IBF (sIBF). Secondly, the subspace problem is solved using a regularised or Bayesian estimator. In the EM-IBF solution, the sIBF act like a low-pass spatial filter: They impose the minimum smoothness the solution should have. Any high spatial frequency activity is treated as noise.

Classical approaches to noise regularisation for distributed linear solutions are usually empirical and proceed on a trial-and-error basis: The level of regularisation is adapted manually such that the ensuing solution and assumed noise component seem reasonable. In contrast, the ReML procedure was successfully applied to control the noise regularisation by systematically estimating a regularisation hyperparameters. With noise regularisation, the IBF, MS and WMN solutions behaved almost as they did with noise-free data. With no noise regularisation, the MS solution was affected strongly by noise and was unable to provide any proper localisation, even for a high SNR. The maximum smoothing constraint alone is thus not enough to discount the effect of the noise.

Even at constant SNR, the values of the hyperparameters vary over a wide range. As the noise component $\varepsilon$ was similar throughout the simulations (simple white noise), the value of the hyperparameters depends on the source configuration and the distribution of potentials it generates over the scalp. Therefore, any fixed value of the hyperparameters can lead to suboptimal solutions. This is an important point and a fundamental motivation

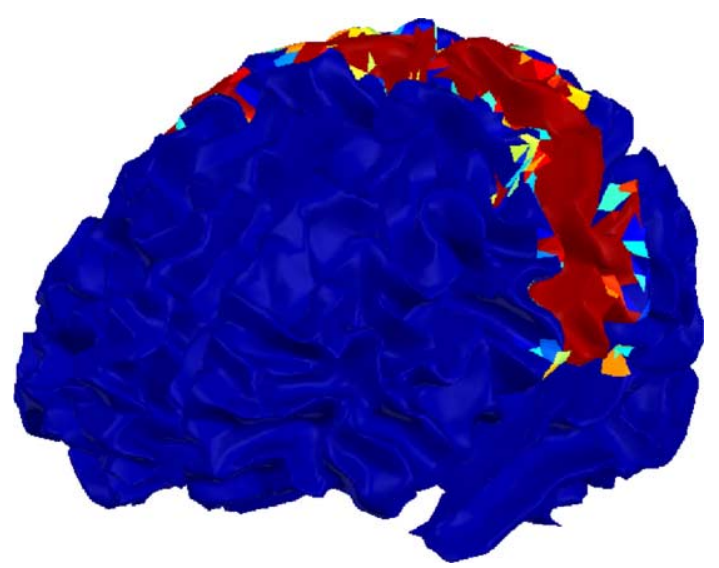

Fig. 10. Location prior used to constrain the source reconstruction. Note that the prior is encompasses the whole area around the post-central gyrus, on both hemispheres.

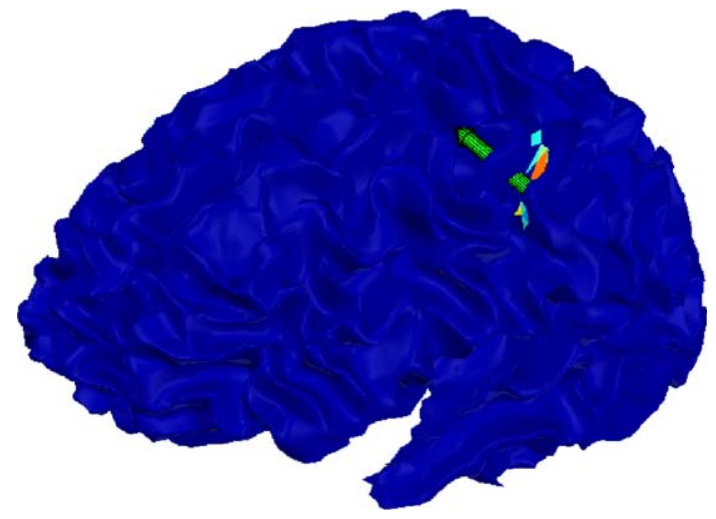

Fig. 11. Source reconstruction (norm of the sources thresholded at $2 / 3$ of the maximum and normalised between 0 and 1) with the location prior constraints showed in Fig. 10. The yellow arrow shows more clearly the location and orientation of the source reconstruction maximum.

for the adaptive ReML estimates proposed here. For example, some sources may arise in cortical regions where priors can be specified very precisely leading to high values of $v_{\mathrm{i}}$. In other regions, priors may be less informative rendering a smaller value $v_{i}$ more appropriate. The flexibility afforded by parametrising the priors in terms of hyperparameters lies in being able to specify the components of the covariances $\left(\mathbf{C}_{\varepsilon}\right.$ and $\left.\mathbf{C}_{\mathbf{j}}\right)$ without fixing their relative contributions. These contributions are scaled by the hyperparameters that we estimated by ReML. The advantage of this approach is that the relative importance of the likelihood of, and priors on, the solution can be determined empirically. This affords the prior constraints the latitude to shape themselves in relation to observation error and each other. One must still be aware that one cannot assess the intrinsic quality of the priors used from the solution obtained, i.e., if only inaccurate or incomplete priors (of any kind) are used, the solution can be inaccurate.

With a realistic head model and an extracted cortical surface based on the subject's structural MRI, errors still arise when estimating the lead field even though the model is anatomically more accurate. One source of error is the constraint imposed on the source orientation. If not perfectly accurate, the extracted cortical surface should be used as an approximation of the real source orientation. The approach adopted in this paper is an appropriate compromise between orientation-free and orientation-fixed sources.

\section{Appendix A. A Restricted maximum likelihood solution}

\section{A.1. Maximum likelihood solution}

Consider a linear stochastic model of the form

$\mathbf{y}=\mathbf{A x}+\mathbf{r}$

where $\mathbf{A}$, the model or design matrix, is of size $m \times n ; \mathbf{x}$, the unknown vector, is of size $n \times 1 ; \mathbf{y}$, the data vector, and $\mathbf{r}$, the residual or error vector, are of size $m \times 1$ and $\mathbf{C}$, the covariance matrix of $\mathbf{r}=\mathcal{N}(0, \mathbf{C})$, is of size $m \times m$. The normal equations of this model are:

$$
\left(\mathbf{A}^{t} \mathbf{C}^{-1} \mathbf{A}\right) \mathbf{x}=\mathbf{A}^{t} \mathbf{C}^{-1} \mathbf{y}
$$

By solving the normal equations, the "Best Linear Unbiased Estimate" (BLUE) is obtained: 
$\hat{\mathbf{x}}=\left(\mathbf{A}^{t} \mathbf{C}^{-1} \mathbf{A}\right)^{-} \mathbf{A}^{t} \mathbf{C}^{-1} \mathbf{y}$

where, for any matrix $B, B^{-}$denotes an arbitrary generalised inverse of $B$, i.e., any solution to $B B^{-} B=B$. This is equivalent to maximising the following objective likelihood function (Patterson and Thompson, 1971):

$p(\mathbf{y} \mid \mathbf{x}) \propto|\mathbf{C}|^{-1 / 2} e^{-\frac{1}{2}(\mathbf{y}-\mathbf{A x})^{t} \mathbf{C}^{-1}(\mathbf{y}-\mathbf{A x})}$

which is equivalent to maximising the function

$$
\begin{aligned}
F(\mathbf{y} ; \mathbf{x})=\log (p(\mathbf{y} \mid \mathbf{x}))= & -\frac{1}{2} \log |\mathbf{C}| \\
& -\frac{1}{2}(\mathbf{y}-\mathbf{A x})^{t} \mathbf{C}^{-1}(\mathbf{y}-\mathbf{A x}) \\
& + \text { const }
\end{aligned}
$$

The variance-covariance matrix $\mathbf{C}$ is necessary to weight the observations $\mathbf{y}$ according to their variance and to account for their covariance. There may be a model for this matrix, depending on hyperparameters $\theta=\left[\begin{array}{ll}\theta_{1} & \theta_{2} \ldots\end{array}\right]$, but its exact value is not necessarily known a priori and needs to be estimated as well as $\mathbf{x}$.

\section{A.2. Restricted maximum likelihood (ReML) solution}

Assume that the (co-)variance matrix $\mathbf{C}$ is a function of the unknown hyperparameters $\theta_{i}, \mathbf{C}=\mathbf{C}(\boldsymbol{\theta})$ and $\theta=\left[\theta_{1} \theta_{2} \ldots\right]$. There is a scheme that allows the simultaneous estimation of $\mathbf{x}$ and $\mathbf{C}(\boldsymbol{\theta})$ that properly takes into account the loss of degrees of freedom in the model incurred from estimating $\mathbf{x}$ (Harville, 1977) when $\mathbf{C}(\boldsymbol{\theta})$ is calculated.

Starting estimates are initially assigned to $\theta$. With the current estimate $\hat{\boldsymbol{\theta}}, \hat{\mathbf{x}}$ is estimated by maximising the first likelihood function (29). Then, an updated estimate of $\boldsymbol{\theta}$ is calculated from the current value $\hat{\mathbf{x}}$. The procedure is repeated until convergence of both $\hat{\mathbf{x}}$ and $\hat{\boldsymbol{\theta}}$.

Harville (1974) showed that this iterative procedure maximises the following function that has subsequently been named the "Restricted Maximum Likelihood" (ReML) objective function:

$p(\mathbf{y} \mid \mathbf{x}, \theta)=\sqrt{\frac{\left|\mathbf{A}^{t} \mathbf{A}\right|}{(2 \pi)^{m-n}|\mathbf{C}(\theta)|\left|\mathbf{A}^{t} \mathbf{C}(\theta)^{-1} \mathbf{A}\right|}} e^{-\frac{1}{2}(\mathbf{y}-\mathbf{A} \mathbf{x})^{t} \mathbf{C}(\theta)^{-1}(\mathbf{y}-\mathbf{A x})}$

Maximising Eq. (31) is equivalent to maximising:

$$
\begin{aligned}
F(\mathbf{y} ; \mathbf{x}, \theta)=\log (p(\mathbf{y} \mid \mathbf{x}, \theta))= & -\frac{1}{2} \log \left|\mathbf{A}^{t} \mathbf{C}(\theta)^{-1} \mathbf{A}\right|-\frac{1}{2} \log |\mathbf{C}(\theta)| \\
& -\frac{1}{2}(\mathbf{y}-\mathbf{A} \mathbf{x})^{t} \mathbf{C}(\theta)^{-1}(\mathbf{y}-\mathbf{A x}) \\
& + \text { const }
\end{aligned}
$$

There exists a general iterative approach for estimating $\mathbf{x}$ and $\boldsymbol{\theta}$ with a linear parameterisation of $\mathbf{C}$, i.e., $\mathbf{C}(\boldsymbol{\theta})=\sum \theta_{i} \mathbf{G}_{i}$, where $\mathbf{G}_{i}$ are $n \times n$ symmetric matrices whose elements are known. This leads to a simple and computationally expedient approach (Harville, 1977), which is formally identical to "expectation maximisation" (Friston et al., 2002).

\section{Appendix B. Expectation-maximisation algorithm}

This second appendix is a summary of the treatment presented in Friston et al. (2002).

\section{B.1. General EM algorithm}

The objective of an Expectation-Maximisation (EM) algorithm is to maximise the likelihood $p(y \mid \theta)$ of the observed data $y$, conditional on some hyperparameters $y$, in the presence of unobserved variables $x$ :

$p(y \mid \theta)=\int_{x} p(x, y \mid \theta) \mathrm{d} x$

A distribution $Q(x)$ is introduced to approximate $p(x \mid y, \theta)$. The EM algorithm then maximises a lower bound on $p(y \mid \theta)$. The objective function to maximise becomes (the free energy) $\mathcal{F}(Q, \theta)$

$$
\begin{aligned}
\log p(y \mid \theta) \geq \mathcal{F}(Q, \theta)= & \log p(y \mid \theta) \\
& -\int_{x} Q(x) \log \left(\frac{Q(x)}{p(x \mid y, \theta)}\right) \mathrm{d} x \\
& =\int_{x} Q(x) p(x \mid y, \theta) \mathrm{d} x-\int_{x} Q(x) \log Q(x) \mathrm{d} x
\end{aligned}
$$

The EM algorithm maximises $\mathcal{F}(Q, \theta)$ by alternating the "Estep" and the "M-step":

- E-step: maximise $\mathcal{F}(Q, \theta)$ with respect to $Q(x)$, while keeping $\theta$ constant.

- M-step: maximise $\mathcal{F}(Q, \theta)$ with respect to $\theta$, while keeping $Q(x)$ constant.

The maximum in the E-step is obtained when actually $Q(x)=$ $p(x \mid y, \theta)$, at which point, Eqs. (34a) and (34b) becomes an equality. The M-step finds the "Maximum Likelihood" (ML) estimate of the hyperparameters, i.e., the values of $\theta$ that maximise $p(y \mid \theta)$ integrating $p(x, y \mid \theta)$ over the parameters using the current estimate of their conditional distribution.

\section{B.2. EM and hierarchical model}

In the case of a 2-level hierarchical model, like the source localisation problem expressed by Eq. (13) in "Hierarchical parametric empirical Bayes approach".

$\mathbf{y}=\mathbf{A}^{(1)} \mathbf{x}^{(1)}+\varepsilon^{(1)}$

$\mathbf{x}^{(1)}=\mathbf{A}^{(2)} \mathbf{x}^{(2)}+\varepsilon^{(2)}$

under Gaussian assumptions about the errors $\varepsilon^{(i)} \propto \mathcal{N}\left(0, \mathbf{C}_{\varepsilon}^{(i)}\right)$, where each covariance matrix $\mathbf{C}_{\varepsilon}^{(1)}$ is defined as a function of some hyper parameters $\theta=\left[\begin{array}{lll}\theta_{1} & \theta_{2} \ldots \theta_{n}\end{array}\right]^{t}$. By substituting Eq. (35b) into Eq. (35a), only the final level parameters are left in addition to 
random effects :

$\mathbf{y}=\varepsilon^{(1)}+\mathbf{A}^{(1)} \varepsilon^{(2)}+\mathbf{A}^{(1)} \mathbf{A}^{(2)} \mathbf{x}^{2}$

The covariance partitioning implied by Eq. (36) is

$E\left\{\mathbf{y} \mathbf{y}^{t}\right\}=\underbrace{\mathbf{C}_{\varepsilon}^{(1)}}_{\text {error }}+\underbrace{\mathbf{A}^{(1)} \mathbf{C} \varepsilon^{(2)} \mathbf{A}^{(1) t}}_{\text {random effect }}+\underbrace{\mathbf{A}^{(1)} \mathbf{A}^{(2)} \mathbf{x}^{(2)} \mathbf{x}^{(2) t} \mathbf{A}^{(2) t} \mathbf{A}^{(1) t}}_{\text {fixed effect }}$

In this hierarchical model, the E-step consists in taking the conditional (or posterior) mean and covariance of the unknown parameters:

$\mathbf{C}_{\left.x\right|_{\hat{y}}}=\left(\overline{\mathbf{A}}^{t} \mathbf{C}_{\varepsilon}^{-1} \overline{\mathbf{A}}\right)^{-1}$

$\eta_{\left.x\right|_{\hat{y}}}=\mathbf{C}_{\left.x\right|_{\hat{y}}}\left(\overline{\mathbf{A}}^{t} \mathbf{C}_{\varepsilon}^{-1} \overline{\mathbf{y}}\right)$

where $\overline{\mathbf{y}}=\left[\begin{array}{c}\mathbf{y} \\ 0 \\ \eta_{\mathbf{x}}^{(2)}\end{array}\right], \overline{\mathbf{A}}=\left[\begin{array}{cc}\mathbf{A}^{(1)} & \mathbf{A}^{(1)} \mathbf{A}^{(2)} \\ \mathbf{I} & 0 \\ 0 & \mathbf{I}\end{array}\right]$

and $\mathbf{C}_{\varepsilon}=\left[\begin{array}{ccc}\mathbf{C}_{\varepsilon}^{(1)} & 0 & 0 \\ 0 & \mathbf{C}_{\varepsilon}^{(2)} & 0 \\ 0 & 0 & \mathbf{C}_{\mathbf{x}}^{(2)}\end{array}\right]$

as expressed in Friston et al. (2002, Section 2.3).

The covariance matrix $\mathbf{C}_{\varepsilon}$ is a linear combination of variance components such that $\mathbf{C}_{\varepsilon}=\sum \theta_{i} \mathbf{Q}_{i}$. Note that Eqs. (38a) and (38b) corresponds formally to Eqs. (8a) and (8b). In our application, $\eta_{\mathbf{x}}^{(2)}=0$ and $\mathbf{C}_{\mathbf{x}}^{(2)}=\infty$, such that $\mathbf{C}_{\mathbf{x}}^{(2)-1} \eta_{\mathbf{x}}^{(2)}=0$.

For the M-step, only the hyperparameters $\boldsymbol{\theta}$ of the errors covariances $\mathbf{C}_{\varepsilon}^{(i)}$ need to be estimated. Specifically, we seek the hyperparameters that maximise the first term in the expression (34b) for $\mathcal{F}(Q, \theta)$. Given the assumptions made in expressions (35a) and (35b), $\mathcal{F}$ is expressed by

$$
\begin{aligned}
\mathcal{F}= & \frac{1}{2} \log \left|\mathbf{C}_{\varepsilon}^{-1}\right|-\frac{1}{2} \mathbf{r}^{t} \mathbf{C}_{\varepsilon}^{-1} \mathbf{r} \\
& -\frac{1}{2} \operatorname{trace}\left\{\mathbf{C}_{\left.x\right|_{y}} \overline{\mathbf{A}}^{t} \mathbf{C}_{\varepsilon}^{-1} \overline{\mathbf{A}}\right\}+\frac{1}{2} \log \left|\mathbf{C}_{\left.x\right|_{y}}\right|+\text { const }
\end{aligned}
$$

where the residuals $\mathbf{r}=\overline{\mathrm{y}}-\overline{\mathrm{A}} \eta_{\left.x\right|_{\hat{y}}}$. The derivatives of $\mathcal{F}$ with respect to the hyperparameters $\theta_{i}$ can be calculated and the maximum found (Dempster et al., 1981; Friston et al., 2002, Appendix 1).

\section{B.3. Relationship to ReML}

Under the present assumptions ReML is formally identical to EM. One can regard ReML as embedding the E-step into the Mstep to provide a single log-likelihood objective function: substituting the conditional covariance $\left.\mathbf{C}_{\mathrm{x}}\right|_{\hat{y}}$ from Eq. (38a) into Eq. (39) gives the ReML objective function. Operationally, the derivatives of $\mathcal{F}$ with respect to the hyperparameters $\theta_{i}$ can be rearranged to give a ReML scheme by removing any explicit reference to the conditional covariance (Harville, 1977; Friston et al., 2002, Appendix 2).

$\theta \leftarrow \theta+\mathbf{H}^{-1} \mathbf{g}$

$g_{i}=-\frac{1}{2} \operatorname{trace}\left\{\mathbf{P} \mathbf{Q}_{i}\right\}+\frac{1}{2} \operatorname{trace}\left\{\mathbf{P} \overline{\mathbf{y y}}^{t} \mathbf{P}^{t} \mathbf{Q}_{1}\right\}$
$H_{i j}=\frac{1}{2} \operatorname{trace}\left\{\mathbf{P} \mathbf{Q}_{i} \mathbf{P} \mathbf{Q}_{j}\right\}$

$\mathbf{P}=\mathbf{C}_{\varepsilon}^{-1}-\mathbf{C}_{\varepsilon}^{-1} \overline{\mathbf{A}}\left(\overline{\mathbf{A}}^{t} \mathbf{C}_{\varepsilon}^{-1} \overline{\mathbf{A}}\right)^{-1} \overline{\mathbf{A}}^{t} \mathbf{C}_{\varepsilon}^{-1}$

The particular form of Eqs. (40a) (40b) (40c) (40d) has a very useful application when $\mathbf{y}$ is a multivariate data matrix and the hyperparameters are the same for all columns, i.e., in the case of EEG source reconstruction, over a window of time. The time-wide hyperparameters can thus be obtained efficiently by iterating Eqs. (40a) (40b) (40c) (40d) using the sample covariance matrix $\mathbf{C}_{y}=\mathbf{y} \mathbf{y}^{t}$. This algorithm is used in the current version of the SPM software (Wellcome Department of Cognitive Neurology, 2002 http://www. fil.ion.ucl.ac.uk/spm).

\section{References}

Aine, C., Huang, M., Stephen, J., Christner, R., 2000. Multistart algorithms for MEG empirical data analysis reliably characterize locations and time courses of multiple sources. NeuroImage 12 (2), 159-172.

Ary, J.P., Klein, S.A., Fender, D.H., 1981. Location of sources of evoked scalp potentials: corrections for skull and scalp thickness. IEEE Trans. Biomed. Eng. 28 (6), 447-452.

Backus, G.E., Gilbert, J.F., 1970. Uniqueness in the inversion of inaccurate gross earth data. Philos. Trans. R. Soc. 266, 123-192.

Brooks, D.H., Ahmad, G.F., MacLeod, R.S., Maratos, G.M., 1999. Inverse electrocardiography by simultaneous imposition of multiple constraints. IEEE Trans. Biomed. Eng. 46, 3-17.

Cuffin, B.N., Cohen, D., 1979. Comparison of the magnetoencephalogram and electroencephalogram. Electroencephalogr. Clin. Neurophysiol. 47, $132-146$.

Dempster, A.P., Rubin, D.B, Tsutakawa, R.K., 1981. Estimation in covariance component models. J. Am. Stat. Assoc., 341-353.

Desmedt, J.E., Cheron, G., 1981. Non-cephalic reference recording of early somatosensory potentials to finger stimulation in adult or aging normal man: differentiation of widespread N18 and contralateral N20 from the prerolandic P22 and N30 components. Electroencephalogr. Clin. Neurophysiol. 52 (6), 553-570.

Friston, K.J., Penny, W., Phillips, C., Kiebel, S., Hinton, G.E., Ashburner, J., 2002. Classical and Bayesian inference in neuroimaging: theory. NeuroImage 16, 465-483.

Gonzalez Andino, S.L., Blanke, O., Lantz, G., Thut, G., Grave de Peralta Menendez, R., 2001. The use of functional constraints for the neuroelectromagnetic inverse problem: Alternatives and caveats. Int. J. Bioelectromagn. 3 (1).

Grave de Peralta Menendez, R., Gonzalez Andino, S., 1998. A critical analysis of linear inverse solutions to the neuroelectromagnetic inverse problem. IEEE Trans. Biomed. Eng. 45 (4), 440-448.

Grave de Peralta Menendez, R., Gonzalez Andino, S., 1999. Backus and Gilbert method for vector fields. Hum. Brain Mapp. 7, 161-165.

Hämäläinen, M.S., Ilmoniemi, R.J., 1994. Interpreting magnetic fields of the brain: minimum norm estimates. Med. Biol. Eng. Comput. 32, 35-42.

Hansen, P.C., 1992. Analysis of discrete Ill-posed problems by means of the L-curve. SIAM Rev. 34, 561-580.

Harville, D.A., 1974. Bayesian inference for variance components using only error contrasts. Biometrika 61, 383-385.

Harville, D.A., 1977. Maximum likelihood approaches to variance component estimation and to related problems. J. Am. Stat. Assoc. 72, 320-338 (June).

Ioannides, A.A., Bolton, J.P.R., Clarke, C.J.S., 1990. Continuous probabilistic solutions to the biomagnetic inverse problem. Inverse Probl. 6 (4), 523-543.

Maldjian, J.A., Laurienti, P.J., Kraft, R.A., Burdette, J.H., 2003. An 
automated method for neuroanatomic and cytoarchitectonic atlas-based interrogation of fmri data sets. NeuroImage 19, 1233-1239 (WFU Pickatlas, version 1.03).

Miltner, W., Braun, C., Johnson, R., Simpson, G.V., Ruchkni, D.S., 1994. A test of brain electrical source analysis (BESA): a simulation study. Electroencephalogr. Clin. Neurophysiol. 91, 295-310.

Nunez, P.L., 1981. Electric Fields of the Brain: The Neurophysics of EEG. Oxford Univ. Press, New York.

Pascual-Marqui, R.D., 1995. Reply to comments by M. Hämäläinen, R. Ilmoniemi and P. Nunez. In: Skrandies, W. (Ed.), Source Localization: Continuing Discussion of the Inverse Prolem, ISBET Newsl., vol. 6. pp. $16-28$.

Pascual-Marqui, R.D., 1998. Low Resolution Brain Electromagnetic Tomography (LORETA). http://www.unizh.ch/keyinst/NewLORETA/ LORETA01.htm.

Pascual-Marqui, R.D., 1999. Review of methods for solving the EEG inverse problem. Int. J. Bioelectromagn. 1 (1), 75-86.

Pascual-Marqui, R.D., Michel, C.M., Lehmann, D., 1994. Low resolution electromagnetic tomography: a new method for localizing electrical activity in the brain. Int. J. Psychophysiol. 18, 49-65.

Patterson, H.D., Thompson, R., 1971. Recovery of inter-block information when block sizes are unequal. Biometrika 58, 545-554.

Perrin, F., Pernier, J., Bertrand, O., Echallier, J.F., 1989. Spherical splines for scalp potential and current density mapping. Electroencephalogr. Clin. Neurophysiol. 72, 184-187.

Phillips, C., Rugg, M.D., Friston, K.J., 2002a. Anatomically informed basis functions for EEG source localization: combining functional and anatomical constraints. NeuroImage 16, 678-695.

Phillips, C., Rugg, M.D., Friston, K.J., 2002b. Systematic regularization of linear inverse solutions of the EEG source localization problem. NeuroImage 17, 287-301.

Polhemus Fastrak. 2003. 40 Hercules Dr., P.O. Box 560, Colchester, VT 05446, USA. http://www.polhemus.com.

Rivière, D., Papadopoulos-Orfanos, D., Régis, J., Mangin, J.-F., 2000. A structural browser of brain anatomy. NeuroImage 11 (8).

Rivière, D., Papadopoulos-Orfanos, D., 2003. BrainVisa. http://brainvisa. info.

Sarvas, J., 1987. Basic mathematical and electromagnetic concepts of the biomagnetic inverse problem. Phys. Med. Biol. 32, 11-22.

Scherg, M., Ebersole, J.S., 1994. Brain source imaging of focal and multifocal epileptiform EEG activity. Clin. Neurophysiol. 24, 51-60.

Scherg, M., Bast, T., Berg, P., 1999. Multiple source analysis of interictal spikes: goals, requirements, and clinical value. J. Clin. Neurophysiol. 16 (3), 214-224.

Spinelli, L., Gonzalez Andino, S., Lantz, G., Seeck, M., Michel, C.M., 2000. Electromagnetic inverse solutions in anatomically constrained spherical head models. Brain Topogr. 13 (2), 115-125.

Tikhonov, A.N., Arsenin, V.Y., 1977. Solutions of Ill-Posed Problems. John Wiley, New York.

Uutela, K., Hämäläinen, M.S., Somersalo, E., 1999. Visualization of magnetoencephalographic data using minimum current estimates. NeuroImage $10,173-180$

von Helmholtz, Hermann, L.F., 1853. Ueber einige Gesetze der Vertheilung elektrischer Ströme in köperlichen Leitern mit Anwendung auf die thierisch-elektrischen Versuche. Annalen der Physik und Chemie, 89, 211-233, 354-377.

Wellcome Department of Cognitive Neurology, 2002. http://www.fil.ion. ucl.ac.uk/spm. Statistical Parametric Mapping, SPM2. 